\title{
Potentials on the Two-Torus for Which the Hamiltonian Flow is Ergodic
}

\author{
Victor Donnay $^{1 \star}$ and Carlangelo Liverani ${ }^{2,3}$ \\ 1 Mathematics Department, Bryn Mawr College, Bryn Mawr, PA 19010, USA \\ ${ }^{2}$ Mathematics Department, University of Arizona, Tucson, AZ 85721, USA \\ 3 Mathematics Department, University of Rome II, Italy
}

Received March 25, 1990

\begin{abstract}
We consider the motion of a test particle in a compound central potential field on a two-dimensional torus. We discuss three different classes of potentials (attracting, repelliny, and mixed) that lead to Hamiltonian systems which have positive Lyapunov exponent almost everywhere and are ergodic. Included among the mixed potentials are smooth potentials without singularities.
\end{abstract}

\section{Introduction}

Do gas molecules interacting in a box behave stochastically? Boltzmann's ergodic hypothesis, rephrased in modern language, asserts the affirmative. In the nineteen forties, the Russian physicist Krylov [Kr] studied the case of a gas of hard spheres. His calculations indicated that collisions between the spheres would lead to an exponential divergence of trajectories. Hopf $[\mathrm{H}]$ had recently shown that geodesic flow on surfaces of negative curvature was ergodic precisely because of such exponential instability of trajectories. Krylov argued that the hard sphere gas should therefore also behave stochastically.

In the early 1960s, Sinai [Si1] continued the work of Krylov. He translated the problem of two hard spheres into a billiard system consisting of one particle moving on a two dimensional torus $T^{2}$ with circular obstacles (scatterers). Sinai [Si2] showed that this billiard system was ergodic. Furthermore, recent work has proven ergodicity of systems of three and four balls [SC, KSS1, KSS2].

We will study a related systems, also discussed by Krylov, obtained by replacing the circular scatters on the billiard table by symmetric potentials of finite range. One then examines the motion of a point particle in the potential field. The resulting

* Partially supported by NSF grant DMS 8806067 
dynamical system is determined by a Hamiltonian function $H: \frac{d p}{d t}=\frac{\partial H}{\partial q}, \frac{d q}{d t}=-\frac{\partial H}{\partial p}$. If there are $n$ disjoint scatterers on the torus, then letting $p \in R^{2}, q \in T^{2}$,

$$
H(p, q)=\frac{1}{2} p^{2}+\sum_{i=1}^{n} V_{i}\left(q-q_{i}\right)
$$

where the radially symmetric potentials $V_{i}$ vanish outside a disk $D_{i}$ of radius $R_{i}$.

The total energy $E$ of the point particle is preserved under the Hamiltonian flow induced by (1.1) We restrict our attention to the $E=1 / 2$ energy surface; a compact three dimensional manifold which we denote by $\mathscr{M}$. The choice of $E=1 / 2$ implies that the particle will move with unit speed while outside $\cup D_{i}$. We denote by $\phi^{t}$ the flow induced on $\mathscr{M}$ by (1.1) and let $\mu$ be the restriction of the Louville measure to $\mathscr{M}$. This measure is invariant under $\phi^{t}$. We will study the dynamical system

$$
\left(\mathscr{M}, \phi^{t}, \mu\right) \text {. }
$$

To simplify matters, we will discuss the case of one scatterer and will set $q_{1}$ equal to the origin, but our results apply to the more general situation as well.

Recently, Knauf [Kn1] examined the case of attracting potentials and showed the existence of attracting potentials with $-r^{-1}$ singularities (Coulomb), and more generally singularities of type $-r^{-2(1-1 / n)}, n \in Z^{+} \backslash\{0,1\}$, for which the system has positive entropy and is ergodic. We generalize this result to show

Theorem 1. For any $\alpha \in(0,2)$, there exist attracting potentials with singularity of order $-r^{-\alpha}$ for which the system has positive Lyapunov exponents almost everywhere and is ergodic.

Note that if the singularity is $-r^{-2}$ or stronger, then a positive measure set of trajectories would be pulled into the singularity, trivializing the dynamics.

In the case of repelling potentials, Sinai [Si1] and then later Kubo [Ku] showed

Theorem 2. There exist repelling potentials, which are continuous but not $C^{1}$, for which the system has positive Lyapunov exponent almost everywhere and is ergodic.

In their examples, the discontinuity in $V^{\prime}$ occurred at the boundary of the disk. At the discontinuity, they needed that $\left|V^{\prime}\right|$ was greater than a certain energy dependent lower bound. We generalize their results by constructing potentials with an arbitrarily small discontinuity in $V^{\prime}$ for which the conclusion of Theorem 2 still holds. Other works on this subject are [KSS4, KuM, M, V1, V2].

In [D1, D2], Donnay created smooth metrices on the sphere and two-torus for which the geodesic flow had positive Lyapunov exponent almost everywhere and was ergodic (see also [BG2]). In analogy with his construction, we show

Theorem 3. There exist smooth potentials without singularities for which the system has positive Lyapunov exponent almost everywhere and is ergodic.

These are the first examples of a smooth Hamiltonian of the form $H=$ Kinetic Energy + Potential Energy for which the flow is ergodic [Be, p. 185].

We can construct several different sorts of smooth potentials. The crucial ingredient in the construction is that there exist a closed orbit inside the disk $D$.

We prove our results on Lyapunov exponents by applying Wojtkowski's 


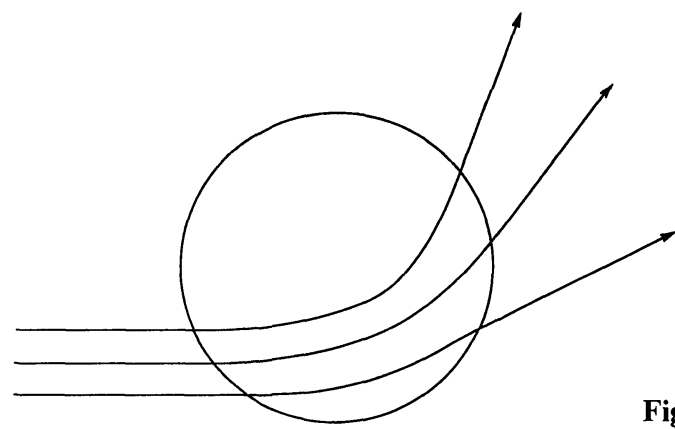

Fig. 1. Attracting potential causes divergence

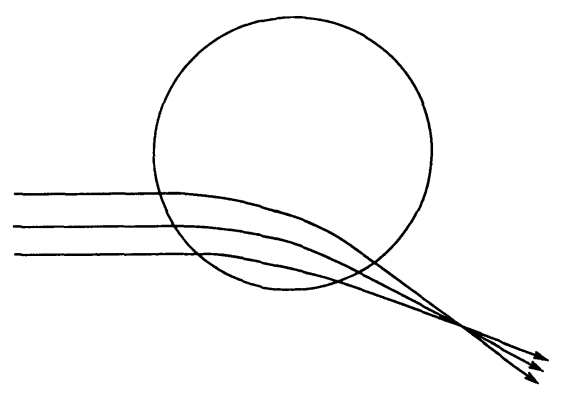

Fig. 2. Repelling potential causes focusing

method of invariant cone-fields [W1] to the flow $\phi^{t}$. A positive Lyapunov exponent implies that a family of infinitesimally nearby trajectories will diverges from one another exponentially fast.

In free motion, $V \equiv 0$, a parallel family of trajectories stays parallel and never diverges. The attracting potentials of Theorem 1 will cause every parallel family of trajectories that enters the disk to become strictly divergent (Fig. 1) Once all parallel families are shown to become strictly divergent, the cone-field method immediately implies that almost every such family will diverge exponentially.

The repelling potentials of Theorem 2 cause the parallel family to converge. Before returning to the next potential, the converging family focuses and then becomes strictly divergent (Fig. 2). If the repelling potential were $C^{1}$ smooth, then the time until this focusing occurred could become arbitrarily large; in particular longer than the return time to the potential. The method of proof then breaks down.

For the systems of Theorem 3, the trajectories that enter the disk but do not cross the closed orbit will exhibit the diverging behavior. Those trajectories that cross the closed orbit will display, in a finite time, the focusing behavior. Dividing these two sets of trajectories are the trajectories that become asymptotic to the closed orbit.

These trajectories introduce a type of discontinuity into the system. Typically, in 
going from the diverging behavior, to the focusing-in-finite-time behavior, one must encounter the focusing-in-unbounded-time behavior. We are able to skip over this intermediate situation because of the discontinuity caused by the closed orbit.

To prove ergodicity, two different methods are available. One method is based on the Burns-Gerber [BG1] argument for contact flows. The Burns-Gerber argument relies heavily on Pesin theory [P] and requires that the system be smooth. This smoothness requirement fails for the examples of Theorems 1 and 2. However for the special potentials with singularities of order $-r^{-2(1-1 / n)}$, we can regularize the singularity to produce a smooth flow. We combine this regularization with the cone technique to give a different proof of Knauf's ergodicity result.

Knauf's proof was based on the Maupertius principle [A, p. 246] which states that the motion of a particle under the effect of a potential can be viewed as geodesic motion relative to a special metric. The trajectories on the torus produced by the potential $V$ agree with the geodesic trajectories produced by the Riemannian metric

$$
d s^{2}=(E-V(q)) d \tilde{s}^{2},
$$

where $d \tilde{s}$ is the Euclidean metric and $E$ is the total energy of the particle. Note that although the trajectories of the two systems coincide, the time parametrizations of the flows do not. For any examples in Theorem 3 one can prove ergodicity by combining the Maupertius principle with the Burns-Gerber argument.

For the potentials he constructed, Knauf showed that inside the disk $D$, the metric $d s$ was of negative curvature. Outside the disk the curvature is clearly zero. For such a metric, the geodesic flow is ergodic (in an appropriate covering space) and hence so is the potential flow. We note that Krylov [Kr] did calculations for potentials of power-type indicating the likelihood that the associated metrics would have negative curvature.

The other method is based on Chernov's and Sinai's ideas [SC]. These techniques were developed to prove ergodicity in the case of non-smooth systems (systems of hard balls), and relied on a detailed analysis of the properties of the singularities in the system.

Finally in analogue with Donnay's light-bulb example [D1], we show

Theorem 4. There exist smooth potentials without singularities for which the system has positive Lypunov exponent on a set of positive measure but is not ergodic.

The outline of our paper is as follows. We review (Sect. 2) the necessary material about cone-fields. We then consider the potential system as an example of a more general abstract system (Sect. 3). We determine conditions for this abstract system that will insure positive Lyapunov exponents (Sect. 4). We use these conditions to prove Theorems 1, 2, 3 and 4 (Sect. 5). We discuss the ergodicity of our examples (Sect. 6). We relate (Sect. 7) our potentials to the metrics given by the Maupertius principle. In Appendices I, II, we compute explicitly some relevant quantities for our model, and obtain the estimates needed to prove the existence of the Lyapunov exponents for the flows under consideration. In Appendix III, we supply calculations used for the proof of Theorem 3. In Appendix IV, we provide the modifications necessary to apply the Sinai-Chernov theory, in the version presented in [KSS3], to our examples. Finally, Appendix V discusses embedding the associated Maupertius metrics. 


\section{Lyapunov Exponents and Cone-Fields}

Let $\mathscr{M}$ be a $C^{3}$ compact, connected, three dimensional Riemannian manifold and $\mu$ a measure on $\mathscr{M}$ that is equivalent to the Riemannian volume. Let $\phi^{t}$ be a measurable flow on $\mathscr{M}$, generated by a vector field $X$, that preserves $\mu$. Osceledec's multiplicative ergodic theorem [O] implies that for a flow satisfying

$$
\int_{\mathscr{M}} \log ^{+} \sup _{t \in[0,1]}\left\|d \phi^{t}(x)\right\| d \mu(x)<\infty,
$$

one has that for $\mu$-a.e. $x \in \mathscr{M}$ the Lyapunov exponents of $\xi \in \mathscr{T}_{x} \mathscr{M}, \xi \neq 0$,

and

$$
\lambda^{+}(x, \xi)=\lim _{t \rightarrow+\infty} \frac{1}{t} \log \left\|d \phi^{t} \xi\right\|
$$

$$
\lambda^{-}(x, \xi)=\lim _{t \rightarrow-\infty} \frac{1}{t} \log \left\|d \phi^{t} \xi\right\|
$$

are well defined.

We define the maximal Lyapunov exponent at $x$ to be

$$
\lambda^{+}(x)=\sup _{\xi \in \mathscr{T}_{x} \mathscr{M}} \lambda^{+}(x, \xi)=\lim _{t \rightarrow \infty} \frac{1}{t} \log \left\|d \phi^{t}(x)\right\| .
$$

Wojtkowski's [W1] technique for proving that the maximal Lyapunov exponent is positive involves cone families.

As in [BG1], we define a cone $C$ in two dimensional vector space $P$ to be a subset $C=C\left(X_{1}, X_{2}\right)=\left\{a X_{1}+b X_{2}: a b \geqq 0\right\}$, where $X_{1}$ and $X_{2}$ are linearly independent vectors and $a, b \in \mathbb{R}$. We call Int $(C)=\left\{a X_{1}+b X_{2}: a b>0\right.$ or $\left.a=b=0\right\}$ the interior of $C$. The family is measurable (continuous) if the vectors $\left\{X_{1}, X_{2}\right\}$ vary in a measurable (continuous) way.

At each point $x \in \mathscr{M}$, we produce a two-dimensional vector space $P(x)$ by taking the three-dimensional tangent space $\mathscr{T}_{x} \mathscr{M}$ and quotienting out by the flow direction $X: P(x)=\mathscr{T}_{x} \mathscr{M} / X(x)$.

For concreteness, we will choose a fixed representative of $P(x)$ at each $x$. We denote by $\mathscr{P}$ the projection in the flow direction onto this subspace $P$; for $\xi=\alpha X+p \in \mathscr{T}_{x} \mathscr{M}, p \in P(x)$, we have

$$
\mathscr{P}(\xi)=p
$$

Theorem 2.1. Assume that the flow satisfies (2.1). Let $U \subset \mathscr{M}$ be a set of positive measure with $X(x) \neq 0$ for every $x \in U$. Suppose that there is a measurable two dimensional distribution $P$ defined on $U$ and a measurable family of cones $C \subset P$ over $U$ such that

(i) $P(x)$ and $X(x)$ span $\mathscr{T}_{x} \mathscr{M}$ for each $x \in U$;

(ii) $\mathscr{P}\left(d \phi^{t} C(x)\right) \leqq C\left(\phi^{t} x\right)$ whenever $x \in U, \phi^{t} x \in U$ and $t \geqq 0$;

(iii) for $\mu$ almost every $x \in U$ there is a $t(x)>0$ such that $\phi^{t(x)} x \in U$ and $\mathscr{P}\left(d \phi^{t(x)} C(x)\right) \subset \operatorname{Int}\left(C\left(\phi^{t(x)} x\right)\right)$.

Then for $\mu$ - a.e. $x \in \hat{U}=\bigcup_{t \in \mathbb{R}} \phi^{t} U$, the maximal Lyapunov exponent satisfies $\lambda^{+}(x)>0$.

Proof. We follow [W1] where the case of maps is studied. Let $T=\phi^{1}$ be the time 
one map of the flow $\phi^{t}$. Let $T_{U}$ be the derived transformation defined for $x \in U$ by $T_{U} x=T^{k_{U}(x)} x$, where $k_{U}(x)=\min \left\{n \geqq 1: T^{n} x \in U\right\}$. We denote by $d T_{U}: U \rightarrow G L(3, R)$ the differential of $T_{U}$. Condition (2.1) implies that $\int_{U} \log ^{+}\left\|d T_{U}(x)\right\| d \mu(x)<\infty$, so that the Lyapunov exponents for the cocycle $\left(T_{U}, d T_{U}\right)$ exist almost everywhere.

Standard ergodic theory techniques imply that if the maximal Lyapunov exponent for the cocycle $\left(T_{U}, d T_{U}\right)$ is positive almost everywhere in $U$, then the same is true for the maximal Lyapunov exponent $\lambda^{+}(x)$ of the flow $\phi^{t}$.

We examine an associated cocycle $\left(T_{U}, \mathscr{P}\left(d T_{U}\right)\right)$, where $\mathscr{P}\left(d T_{U}\right): U \rightarrow G L(2, R)$ is the map from $P(x)$ to $P\left(T_{U} x\right)$ defined using the projection map. The Lyapunov exponents for this cocycle exist since $\log ^{+}\left\|\mathscr{P}\left(d T_{U}\right)\right\| \in L^{1}(U, \mu)$. Conditions (ii) and (iii) allow us to apply [W1, Theorem 2.2] and conclude that the maximal Lyapunov exponent of $\left(T_{U}, \mathscr{P}\left(d T_{U}\right)\right)$ is positive almost everywhere. This result implies that the maximal Lyapunov exponent for $\left(T_{U}, d T_{U}\right)$ is also positive almost everywhere (for more details see also [W2, Lemma 1]).

For smooth flow (at least $C^{2}$ ), Pesin [P] showed that the measure theoretic entropy $h_{\mu}$ is given as the average of the positive Lyapunov exponents.

$$
h_{\mu}=\int_{x \in \mathscr{M}} \lambda^{+}(x) d \mu(x)
$$

Katok and Strelcyn [KS] have extended this result to systems with singularities. If the set $\Gamma$ of points with a positive Lyapunov exponent has positive measure then $\Gamma$ decomposes into at most a countable number of invariant components, each of positive measure. On each component the system exhibits very strong ergodic properties.

Burns and Gerber [BG1] have given a simple condition for ergodicity of smooth systems in terms of cone-fields. Essentially, they show that if the distribution $P$ is flow invariant

$$
d \phi^{t} P(x)=P\left(\phi^{t} x\right)
$$

then continuity of the cone-field implies ergodicity. For systems with singularities, the ergodicity proof of Chernov and Sinai [SC] (see also [KSS3, Bu]) can be phrased in terms of continuous cone-fields, but additional conditions on the singularity manifold are needed. We will refer to these methods to prove ergodicity for our examples (see Sect. 6 and Appendix IV).

\section{Model System}

The dynamics of our system (1.2) consist of the composition of two easily understood motions. Outside the disk, a particle moves in a straight line with unit speed. Inside the disk, the symmetry of the potential implies that the motion is integrable.

To simplify the discussion, we will assume that every particle that starts inside the disk and every particle that enters the disk will leave the disk. This imposes certain restrictions on the potential $V(r)$ (see (3.6)). We give the disk $D$ polar coordinates $(r, \theta), r \in[0, R], \theta \in[0,2 \pi]$, and denote by $\varphi \in[-\pi, \pi]$ the angle a trajectory makes with the boundary of the disk. We set

$$
\begin{aligned}
& \mathscr{S}=\{(\theta, \varphi): \theta \in[0,2 \pi) \text { and } \varphi \in[0, \pi]\}, \\
& \tilde{\mathscr{S}}=\{(\theta, \varphi): \theta \in[0,2 \pi) \text { and } \varphi \in[-\pi, 0]\} .
\end{aligned}
$$




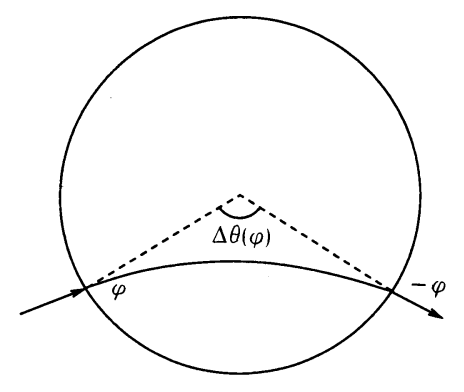

Fig. 3. Rotation function $\Delta \theta(\varphi)$

They represent, respectively, the points in the phase space $\mathscr{M}$ at which trajectories enter and leave the disk. Abusing notation slightly, we will say a point $x=(p, q)$ is in the disk $D$ if $q \in D$.

The symmetry of the potential implies that a particle that enters the disk at the point $(\theta, \varphi), \varphi \in[0, \pi]$, will leave the disk at a point $(\theta+\Delta \theta(\varphi),-\varphi)$ (Fig. 3). The function

$$
\Delta \theta(\varphi), \quad \varphi \in(0, \pi)
$$

is called the rotation function; it will determine the ergodic properties of the system. The exact nature of the rotation function depends on the potential $V(r)$ in the following way.

Let $V(r), r \in \mathbb{R}^{+}$be a radial potential that satisfies

$$
\begin{aligned}
& \lim _{r \rightarrow 0} r^{2} V(r)=0, \\
& \operatorname{supp} V \subset[0, R), \\
& V \in C^{2}((0, R)) .
\end{aligned}
$$

For $r \leqq R$, the trajectory of a particle $(r(t), \theta(t))$ of mass 1 , energy $\frac{1}{2}$, and angular momentum $l$ entering in this potential is given by

$$
\begin{aligned}
\frac{1}{2} & =\frac{1}{2}\left(\dot{r}^{2}+r^{2} \dot{\theta}^{2}\right)+V(r), \\
l & =r^{2} \dot{\theta}, \\
r(0)=R ; \quad \theta(0) & =\theta_{0} \in[0,2 \pi) ; \quad \dot{r}(0) \leqq 0 .
\end{aligned}
$$

The dot indicates the derivative with respect to time and the particle is assumed to enter the disk at time $t=0$.

Rewriting the first of (3.3) gives

where

$$
\dot{r}^{2}=1-\frac{l^{2}}{r^{2}}-2 V(r)=r^{-2}\left(h(r)-l^{2}\right),
$$

$$
h(r)=r^{2}(1-2 V(r)) .
$$

Combining (3.3), (3.4) we have

$$
\frac{d \theta}{d r}= \pm \frac{l}{r \sqrt{h(r)-l^{2}}}
$$


This sign of $\frac{d \theta}{d r}$ depends on whether $r$ is increasing or decreasing.

Before going further we impose an additional condition on our potential,

$$
h^{\prime}(r)>0
$$

for all except perhaps one value of $r \in(0, R)$. This condition insures the absence of "trapping zones": invariant regions of phase space in which the motion is completely integrable (see the end of the section).

With this assumption, if $l \neq 0$, then there exists a time $\bar{t} \in \mathbb{R}^{+} \cup\{\infty\}$ such that $\dot{r}(t) \leqq 0$ for $t<\bar{t}$ and $\dot{r}(\bar{t})=0$. This is the time at which the particle comes closest to the center of the potential. Denote by $\hat{r}=r(\varphi)$ this minimum radius. For potentials satisfying (3.2) and (3.6), we can integrate (3.5) and get the following expression for the rotation function for $\varphi \in[0, \pi / 2)$ :

$$
\Delta \theta(\varphi)=2 \int_{\hat{r}}^{R} \frac{l}{r \sqrt{h(r)-l^{2}}} d r, \quad l=R \cos \varphi=h(\hat{r})^{1 / 2} .
$$

An orbit that enters the potential field with angle $\pi-\varphi$ will rotate clockwise around the disk by the same amount that an orbit entering the disk with angle $\varphi$ will rotate counterclockwise. Thus for $\varphi \in(\pi / 2, \pi]$, we can define

$$
\Delta \theta(\varphi)=-\Delta \theta(\pi-\varphi)
$$

This definition produces a rotation function that will typically be discontinuous at $\varphi=\pi / 2$ (see Lemma 5.5). In such a case, we ignore those trajectories that enter the disk with angle $\varphi=\pi / 2$. These points from a set of measure zero. Note that

$$
\Delta \theta^{\prime}(\varphi)=\Delta \theta^{\prime}(\pi-\varphi),
$$

so that when we study properties that depend only on the derivative of the rotation function, it will suffice to examine $\varphi \in[0, \pi / 2)$.

Actually, in many of the examples, we will be able to define the rotation function to be continuous and even smooth for $\varphi \in[0, \pi]$. If

$$
\lim _{\varphi \rightarrow(\pi / 2)^{-}} \Delta \theta(\varphi)=n \pi, \quad n \in Z,
$$

we set $\Delta \theta(\pi / 2)=n \pi$ and define

$$
\Delta \theta(\varphi)=2 n \pi-\Delta \theta(\pi-\varphi), \quad \varphi \in(\pi / 2, \pi] .
$$

We will derive conditions on the derivative of the rotation function, $\Delta \theta^{\prime}(\varphi)$, which will imply the existence of a strictly invariant cone field. To compute this derivative, we first make the change of variables $s=r / \hat{r}$ to get

$$
\Delta \theta(\varphi)=2 \int_{1}^{R \hat{r}^{-1}} \frac{l}{s \sqrt{h(\hat{r} s)-l^{2}}} d s,
$$

and then differentiate. By trial and error, we have found that the result can be written in a particularly simple form if we introduce the function

$$
\Omega(r)=\frac{r h^{\prime}(r)}{h(r)}=2-2 \frac{V^{\prime}(r) r}{1-2 V(r)} .
$$


One then gets that

$$
\Delta \theta^{\prime}(\varphi)=2 \Omega(\hat{r})^{-1}\left[R^{2}-l^{2}\right]^{1 / 2}\left\{2\left[h\left(R^{-}\right)-l^{2}\right]^{-1 / 2}+\int_{\hat{r}}^{R} \frac{h(r)[\Omega(r)-\Omega(\hat{r})]}{r\left[h(r)-l^{2}\right]^{3 / 2}} d r\right\} .
$$

For details, see Appendix I.

Trapping Zones. If $h^{\prime}(r)<0$ for some $r \in(0, R)$, then there exist orbits that start inside the disk and never leave. Equation (3.4) implies that on a trajectory $(r(t), \theta(t))$, one will always have $h(r(t)) \geqq l^{2}$. Hence trajectories that start inside the disk with a value of angular momentum $l$ close to the maximum value of $h$ will have $r(t) \in\left[r_{\min }, r_{\max }\right]$ for all $t \in \mathbb{R}$, where $h\left(r_{\min }\right)=h\left(r_{\max }\right)=l^{2}$ (Fig. 9).

There can also exist closed orbits inside the disk. If there exist $r_{c} \in(0, R)$ such that

$$
h^{\prime}\left(r_{c}\right)=0 \quad \text { or equivalently } \Omega\left(r_{c}\right)=0,
$$

then there exists a closed orbit with $r(t) \equiv r_{c}$. To see this, let $(r(t), \theta(t))$ be a trajectory with angular momentum $l_{c}^{2}=h\left(r_{c}\right)$ that satisfies $r(0)=r_{c}$. Then using (3.4), we get $\left.\frac{d r}{d t}\right|_{t=0}=0$ and using $h^{\prime}\left(r_{c}\right)=0$ we get $\left.\frac{d^{2} r}{d^{2} t}\right|_{t=0}=0$. Hence $r(t) \equiv r(0)=r_{c}$.

\section{Cone Fields}

We define the cone field $\{C(x)\}, x \in U$, and derive conditions on $\Delta \theta^{\prime}(\varphi)$ that imply the cone-field is strictly invariant (i.e. satisfies (ii, iii) of Theorem 2.1).

Definition 4.1. Let $U \subset \mathscr{M}$ be the set of all points $x=(p, q)$ outside the disk $D$.

We can canonically identify the momentum $p=\dot{q}$ with its velocity vector $\dot{q}$. For $q$ outside the disk, $V(q)=0$, so a particle moves with speed one and the velocity vector $\dot{q}$ has length one. We assign coordinates $\left\{v, v^{\perp}, \Phi\right\}$ to $\mathscr{M}$ in a neighborhood of $x: v$ is the distance from $q$ on $T^{2}$, measured in the direction of $\dot{q}, v^{\perp}$ is the distance from $q$ in the direction perpendicular to $\dot{q}$ and $\Phi$ is the angle of the unit velocity vectors measured counterclockwise relative to a fixed axis. These coordinates induce an orthonormal basis $\left\{X=X_{v}, X_{v^{\perp}}, X_{\Phi}\right\}$ for $T_{x} \mathscr{M}$. We set $P(x)=\operatorname{span}\left\{X_{v^{\perp}}, X_{\Phi}\right\}$.

It is easy to see that as long as $\phi^{t} x$ does not intersect $D$, the distribution $P(x)$ is invariant

$$
d \phi^{t} P(x)=P\left(\phi^{t} x\right)
$$

We define the cone $C(x)$ by

$$
C(x)=\left\{J X_{v^{\perp}}+J^{\prime} X_{\Phi}: J J^{\prime} \geqq 0\right\} .
$$

In differential geometry, a vector $\xi \in P(x)$ is identified with a Jacobi field, hence the notation $\left(J, J^{\prime}\right)$ for the coordinates of the vector.

Theorem 4.2. If for almost every $\varphi \in(0, \pi)$, the rotation function satisfies either $\Delta \theta^{\prime}(\varphi)>2$ or $\Delta \theta^{\prime}(\varphi) \leqq 0$, then the cone family defined by (4.2) is almost everywhere eventually strictly invariant on $U$ (i.e. satisfies (ii, iii) of Theorem 2.1).

We find it useful to interpret vectors $\xi \in \mathscr{T} \mathscr{M}$ geometrically. We identify a vector 
$\xi$ with the one-parameter family of trajectories $\gamma(s)=\left(v(s), v^{\perp}(s), \Phi(s)\right), s \in[-\varepsilon, \varepsilon]$, that generates it: $\gamma(0)=x$ and $\gamma^{\prime}(0)=\xi$. Such a one-parameter family is called a variation. If $\xi \in P(x)$ then $v(s) \equiv 0$ : the variation is perpendicular to the flow direction. The cone specified by (4.2) is then

$$
C(x)=\{\text { variations in } P(x) \text { that are diverging }\} .
$$

The edges of the cone are the variations $\left(J=0, J^{\prime}=1\right)$ and $\left(J=1, J^{\prime}=0\right)$; the former is most strongly divergent, the latter is parallel and is least strongly divergent.

\section{Proof of Theorem 4.2 .}

a. Outside the disk, this family of cones is invariant (i.e. satisfies (ii) of Theorem 2.1): any divergent family of trajectories will stay divergent. The family of cones is not strictly invariant though (does not satisfy (iii) of Theorem 2.1), since the parallel family remains parallel.

b. To understand how the cones evolve when they go through the disk, we examine first the case $V(r) \equiv 0, r \in[0, R]$. Then the trajectories in the disk would be straight lines and by simple trigonometry, one finds that the rotation function is

so that

$$
\Delta \theta(\varphi)=2 \varphi, \quad \varphi \in[0, \pi / 2]
$$

$$
\Delta \theta^{\prime}(\varphi) \equiv 2 \text {. }
$$

The cones would evolve as they had been doing outside the disk. The diverging variations would stay diverging, but the parallel variation would never become strictly divergent; it would remain parallel. Thus the cone field would never become strictly invariant.

To produce a system with positive Lyapunov exponents, we must push the horizontal edge (i.e. the variation $\left(J=1, J^{\prime}=0\right)$ ) of the cone up, so that the cone family becomes strictly invariant. This amounts to making the parallel family become strictly divergent.

c. Knowing the above information in the $V(r) \equiv 0$, case, one can quickly conclude that if

$$
\Delta \theta^{\prime}(\varphi)>2
$$

then a parallel family that enters the disk with angle $\varphi$ will be strictly divergent when it leaves the disk. Also any strictly diverging variation remains strictly divergent. From the geometry of diverging variations one can see that if

$$
\Delta \theta^{\prime}(\varphi) \leqq 0
$$

then the same result holds. A more quantitative proof of this proposition follows from Lemma 4.5.

If the rotation function $\Delta \theta^{\prime}(\varphi)$ takes values in the interval $(0,2)$, then we can still produce an eventually strictly invariant cone family, but we must modify our definition of $C(x)$, and also the minimum time between returns to the disk must be sufficiently large.

For $x \in \mathscr{S}$, we define $t_{0}(x)$ to be the time it takes to go through the disk. We define $t_{1}(x) \in\left(t_{0}(x) .+\infty\right]$ to be the time until $x$ next returns to the disk: $\phi^{t_{1}(x)}(x) \in \mathscr{S}$, if $t_{1}(x)$ is finite. Then for $t \in\left(t_{0}(x), t_{1}(x)\right), \phi^{t} x$ is outside the disk. We set

$$
t_{\min }=\min _{x \in \mathscr{S}}\left(t_{1}(x)-t_{0}(x)\right)
$$


Theorem 4.3. If there exists $a \delta \in(0,2)$ such that $\Delta \theta^{\prime}(\varphi)<2-\delta$ for almost all $\varphi \in[0, \pi]$, then providing that

$$
t_{\min }>\frac{2 R(2-\delta)}{\delta},
$$

there exists a cone-field $C_{1}$ defined on $U$ satisfying conditions (ii) and (iii) of Theorem 2.1

According to the previous theorem a given potential will produce the wanted behavior in a sufficiently large torus. An alternative, but equivalent, point of view is to fix the size of the torus and reduce $R$ by rescaling. In this case (4.9) will be a condition on the support of the potential.

Remark 4.4. If we assume that the torus has width 1 then $t_{\min }=1-2 R$. Condition (4.9) translates into the restriction,

$$
R<\frac{\delta}{4}
$$

To prove this result, we will need more quantitative information about the evolution of variations. Outside the disk, if

$$
\xi=\alpha X+J_{0} X_{v^{\perp}}+J_{0}^{\prime} X_{\Phi} \in \mathscr{T}_{x} \mathscr{M},
$$

then

$$
d \phi^{t} \xi=\alpha X+J(t) X_{v^{\perp}}+J^{\prime}(t) X_{\Phi} \in \mathscr{T}_{\phi_{t} x} \mathscr{M},
$$

where

$$
J(t)=J_{0}+J_{0}^{\prime} t \quad \text { and } \quad J^{\prime}(t)=J_{0}^{\prime} .
$$

For $x=(\theta, \varphi) \in \mathscr{S}$ (see Sect. 3), let $t_{0}(x)$ be the time the orbit takes going through the disk: $d \phi^{t_{0}(x)}=\tilde{x}$, where $\tilde{x} \in \tilde{\mathscr{S}}$. Since the potential is symmetric, the function only depends on $\varphi: t_{0}(x)=t_{0}(\varphi)$.

Lemma 4.5. Let $x=(\theta, \varphi) \in \mathscr{S}$ and

$$
\xi=a X+J X_{v^{\perp}}+J^{\prime} X_{\Phi} \in \mathscr{T}_{x} \mathscr{M} .
$$

Then

$$
d \phi^{t_{0}(\varphi)} \xi=\tilde{\xi}=\tilde{\alpha} X+\tilde{J} X_{v^{\perp}}+\widetilde{J}^{\prime} X_{\Phi} \in \mathscr{T}_{\tilde{x}} \mathscr{M}
$$

where

$$
\begin{aligned}
\tilde{\alpha} & =\alpha+\left(\frac{J}{R \sin \varphi}+J^{\prime}\right)\left(R \Delta \theta^{\prime}(\varphi) \cos \varphi-\frac{d t_{0}}{d \varphi}(\varphi)\right), \\
\tilde{J} & =J\left(\Delta \theta^{\prime}(\varphi)-1\right)+J^{\prime}\left(R \Delta \theta^{\prime}(\varphi) \sin \varphi\right), \\
\tilde{J}^{\prime} & =\frac{J}{R \sin \varphi}\left(\Delta \theta^{\prime}(\varphi)-2\right)+J^{\prime}\left(\Delta \theta^{\prime}(\varphi)-1\right) .
\end{aligned}
$$

The image in $P(\tilde{x})$ of a vector $\xi=J X_{v^{\perp}}+J^{\prime} X_{\Phi} \in \mathscr{T}_{x} \mathscr{M}$, is

$$
\mathscr{P}\left(d \phi^{t_{0}(x)} \xi\right)=\widetilde{J} X_{v^{\perp}}+\widetilde{J}^{\prime} X_{\Phi}
$$


Proof. Let $\gamma(s), s \in(-\varepsilon, \varepsilon)$, be a variation that generates $\xi$ so that $\gamma(0)=x$ and $\gamma^{\prime}(0)=\xi$. We will use a prime for the derivative with respect to $s$ computed at $s=0$ (when it is not conflicting with previous definitions).

a. The tangent space $\mathscr{T} \mathscr{S}$ to the boundary of the disk has basis $\left\{X_{\theta}, X_{\varphi}\right\}$. Since the boundary of the disk has radius of curvature $1 / R$ and remembering that the coordinate $v^{\perp}$ is defined using arc-length, one has that

$$
X_{v^{\perp}}=\cot \varphi X-\frac{1}{R \sin \varphi} X_{\theta}+\frac{1}{R \sin \varphi} X_{\varphi}
$$

Therefore,

$$
\xi=\alpha_{1} X+\theta^{\prime} X_{\theta}+\varphi^{\prime} X_{\varphi}
$$

where

$$
\alpha_{1}=\alpha+J \cot \varphi, \quad \theta^{\prime}=\frac{-J}{R \sin \varphi}, \quad \varphi^{\prime}=\frac{J}{R \sin \varphi}+J^{\prime} .
$$

b. The map from entering the disk to leaving the disk sends $\left(\theta^{\prime}, \varphi^{\prime}\right)$ to $\left(\tilde{\theta}^{\prime}, \tilde{\varphi}^{\prime}\right)$ with

$$
\tilde{\theta}^{\prime}=\theta^{\prime}+\Delta \theta^{\prime}(\varphi) \varphi^{\prime}, \quad \tilde{\varphi}^{\prime}=-\varphi^{\prime}
$$

c. A vector $\tilde{\theta}^{\prime} X_{\theta}+\tilde{\varphi}^{\prime} X_{\varphi} \in \mathscr{T} \tilde{\mathscr{S}}$ can be written as

$$
\tilde{\alpha} X+\tilde{J} X_{v^{\perp}}+\tilde{J}^{\prime} X_{\Phi} \in \mathscr{T} \mathscr{M}
$$

where

$$
\tilde{\alpha}=R \tilde{\theta}^{\prime} \cos \varphi, \quad \tilde{J}=R \tilde{\theta}^{\prime} \sin \varphi, \quad \tilde{J}^{\prime}=\tilde{\theta}^{\prime}+\tilde{\varphi}^{\prime} .
$$

d. Then the composition of the previous three maps gives the vector

$$
\begin{aligned}
\left.\frac{d}{d s} \phi^{t_{0}(\gamma(s))}(\gamma(s))\right|_{s=0} & =\left.\frac{d}{d s} \phi^{t_{0}(\gamma(0))}(\gamma(s))\right|_{s=0}+\left.\frac{d}{d s} t_{0}(\gamma(s))\right|_{s=0} X \\
& =\widetilde{\xi}+\frac{d t_{0}}{d \varphi} \frac{d \varphi}{d s} X .
\end{aligned}
$$

Using (4.16) to determine $\frac{d \varphi}{d s}$, we arrive at (4.12).

In two dimensions, for a vector to be in a cone is projective property: if $\xi \in C(x)$ then $\lambda \xi \in C(x)$ for $\lambda \in \mathbb{R}$. We define a projective coordinate $u$ for the space $P(x)$ : $u=\frac{J^{\prime}}{J}$.

Going through the disk, Lemma 4.5 implies that $\tilde{u}=\frac{\widetilde{J}^{\prime}}{\widetilde{J}}$ is related to $u=\frac{J^{\prime}}{J}$ by

$$
\tilde{u}=f_{\varphi}(u)
$$

where

$$
f_{\varphi}(u)=\frac{\frac{\left(\Delta \theta^{\prime}(\varphi)-2\right)}{R \sin \varphi}+u\left(\Delta \theta^{\prime}(\varphi)-1\right)}{\left(\Delta \theta^{\prime}(\varphi)-1\right)+u\left(R \Delta \theta^{\prime}(\varphi) \sin \varphi\right)}
$$




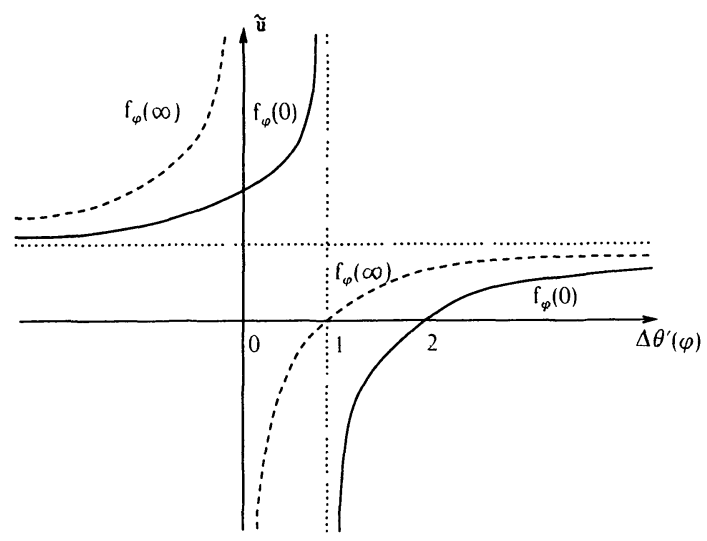

Fig. 4. The effect of going through the disk

The effect of (4.20) on the edges of the cone, $u=0$ and $u=+\infty$, is

$$
\begin{aligned}
f_{\varphi}(0) & =\frac{1}{R \sin \varphi}\left(\frac{\Delta \theta^{\prime}(\varphi)-2}{\Delta \theta^{\prime}(\varphi)-1}\right), \\
f_{\varphi}(\infty) & =\frac{\Delta \theta^{\prime}(\varphi)-1}{R \Delta \theta^{\prime}(\varphi) \sin \varphi} .
\end{aligned}
$$

In Fig. 4, we graph the functions $f_{\varphi}(\infty)$ and $f_{\varphi}(0)$ as functions of $\Delta \theta^{\prime}(\varphi)$. Outside the disk, $u(t)$ evolves according to

$$
u^{\prime}(t)=-u^{2}(t)
$$

We can define global solutions to (4.24): we identify $\pm \infty$ and set $u\left(t_{*}\right)=-\infty$ if $\lim _{t \rightarrow t_{*}} u(t)=-\infty$. For $t>t_{*}$, we continue $u$ so that $\lim _{t \rightarrow t_{*}} u(t)=+\infty$. From (4.11), one immediately gets

Lemma 4.6. If $u(0)<0$, then at time $\tau_{1}=|1 / u(0)|$, one has that $u\left(\tau_{1}\right)=-\infty$. After a further time $\tau_{2}$, one has $u\left(\tau_{1}+\tau_{2}\right)=1 / \tau_{2}$.

Having identified $\pm \infty$, we can consider solutions of (4.24) to live on the circle. The uniqueness property of solutions of ordinary differential equations imply that on the circle, the order of solutions is preserved. If $\tau_{1}<\tau_{2}$ and $u_{0}, u_{1}, u_{2}$ are solutions of (4.24) with $u_{0}\left(\tau_{1}\right)$ between $u_{1}\left(\tau_{1}\right)$ and $u_{2}\left(\tau_{1}\right)$, then $u_{0}\left(\tau_{2}\right)$ is between $u_{1}\left(\tau_{2}\right)$ and $u_{2}\left(\tau_{2}\right)$. The map $f_{\varphi}$ also preserve the ordering.

Proof of Theorem 4.3. For $x=(\theta, \varphi) \in \mathscr{S}$, we define $C_{1}(x)$ by

$$
C_{1}(x)=\left\{J X_{v^{\perp}}+J^{\prime} X_{\Phi}: 0 \leqq J^{\prime} / J \leqq u^{\text {top }}\right\},
$$

where

$$
u^{\text {top }}=\frac{\delta}{R(2-\delta)}
$$


Having defined $\left\{C\left({ }_{1} x\right)\right\}, x \in \mathscr{S}$, we simply push the cones forward to define the cone-field on $U$ : for $\phi^{t}(x) \in U, t \in\left(t_{0}(x), t_{1}(x)\right)$, we define

$$
C_{1}\left(\phi^{t}(x)\right)=\mathscr{P}\left(d \phi_{x}^{t} C_{1}(x)\right) \text {. }
$$

Since almost every $y \in U$ reaches $\mathscr{S}$ in backwards time, (4.27) defines a cone at almost every $y \in U$. Only a set of periodic orbits of measure zero will not reach $\mathscr{S}$. To make the cone field on $U$ strictly invariant, we need only insure that

$$
d \phi^{t_{1}(x)} C_{1}(x) \subset \operatorname{Int}\left(C_{1}\left(\phi^{t_{1}(x)} x\right)\right) \text {. }
$$

By the ordering property of $f_{\varphi}$ and of solutions of (4.24), (4.28) will hold if we show that $u\left(t_{1}(x), x\right)<u^{\text {top }}$, where $u(t, x)$ has initial value $u\left(t_{0}, x\right)=f_{\varphi}\left(u^{\text {top }}\right)$. We claim that for $0<\Delta \theta^{\prime}(\varphi) \leqq 2-\delta$ either $u\left(t_{0}\right)=f_{\varphi}\left(u^{\text {top }}\right) \leqq-\frac{\delta}{R(2-\delta)}$ or $u\left(t_{0}\right)=f_{\varphi}\left(u^{\text {top }}\right) \in$ $(0,+\infty]$. In the first case, after a time $t>\frac{2 R(2-\delta)}{\delta}$ one has that

$$
0<u\left(t+t_{0}\right)<\frac{\delta}{R(2-\delta)}
$$

In the second case, (4.29) holds for $t>\frac{R(2-\delta)}{\delta}$. When $\Delta \theta^{\prime}(\varphi) \leqq 0$ then $0 \leqq u\left(t_{0}\right) \leqq$ $f_{\varphi}(+\infty) \leqq+\infty$ and so (4.29) holds for $t>\frac{R(2-\delta)}{\delta}$.

To prove our claim, we notice that the numerator in (4.21) satisfies

$$
\begin{aligned}
\frac{1}{R \sin \varphi}\left(\Delta \theta^{\prime}(\varphi)-2\right)+u^{\mathrm{top}}\left(\Delta \theta^{\prime}(\varphi)-1\right) & \leqq \frac{-\delta}{R}+u^{\mathrm{top}}(1-\delta) \\
& \leqq \frac{-\delta}{R(2-\delta)}<0
\end{aligned}
$$

and the denominator

$$
\begin{aligned}
\left(\Delta \theta^{\prime}(\varphi)-1\right)+u^{\text {top }}\left(R \Delta \theta^{\prime}(\varphi) \sin \varphi\right) & \leqq(1-\delta)+u^{\text {top }} R(2-\delta) \\
& \leqq 1
\end{aligned}
$$

If $\left(\Delta \theta^{\prime}(\varphi)-1\right)+u^{\text {top }}\left(R \Delta \theta^{\prime}(\varphi) \sin \varphi\right)>0$, then we have the first case.

If $\left(\Delta \theta^{\prime}(\varphi)-1\right)+u^{\text {top }}\left(R \Delta \theta^{\prime}(\varphi) \sin \varphi\right) \leqq 0$, then we have the second case.

Geometrically, our cones consist of variations that the diverging when they first enter $D$ and are again diverging when they return to $D$. If $\Delta \theta^{\prime}(\varphi) \leqq 0$, then any variation that is diverging when it enters $D$ will be diverging when it leaves $D$ and stay diverging until it returns to $D$. For $0<\Delta \theta^{\prime}(\varphi)<2-\delta$, some of the variations will be converging when they leave $D$, but after some finite time they will focus and then be diverging. We insure that they focus before returning to $D$.

Remark 4.7. As $2-\delta$ approaches 2 , the focusing time becomes infinite. So if the value of $\Delta \theta^{\prime}(\varphi)$ goes continuously from 2 to some $2-\varepsilon$, the focusing time for some variation becomes arbitrarily large and our methods of proof breaks down. 
For such system, one would still expect the existence of non-zero Lyapunov exponents. However present methods can not handle this situation in which the expanding direction gets sent onto the contracting direction, in our case by going through the disk. Moreover, in general, it is possible to arrange the geometry of the scatters in such a way as to obtain periodic stable orbits (in the sense of KAM theory), and hence a non-ergodic system. In order to prove positive Lyapunov exponents it would seem necessary to consider a family of similar systems and exclude the ones that may exhibit the undesired behavior. Recent work by [BC] on the Henon map begins to make progress on this type of problems.

\section{Potentials}

For a potential $V(r), r \in(0, R)$ satisfying $V(r) \leqq \frac{1}{2}$, we can use (3.12) to write

$$
V(r)=\frac{1}{2}\left[1-\exp \left(\int_{r}^{R} \frac{2-\Omega(s)}{s} d s\right)\right] .
$$

Since our motion occurs on the $E=\frac{1}{2}$ energy surface, no trajectory can enter the region in which $V>\frac{1}{2}$. Thus any potential we are interested in can be expressed via (5.1) in terms of the corresponding function $\Omega$. This function determines $\Delta \theta^{\prime}(\varphi)$ in a simple way.

\section{Attracting Potentials. We call a potential with $V^{\prime}(r) \geqq 0, r \in(0, R)$, attracting.}

Theorem 5.1. Let $V$ be an attracting potential satisfying (3.2), (3.6) and such that $\Omega(r)$ is strictly increasing for $r \in(0, R)$ with $\Omega(0)>0$ and $V(R)=V^{\prime}(R)=0(\Omega(R)=2)$. Then $\Delta \theta^{\prime}(\varphi)>2$ for all $\varphi \in(0, \pi / 2)$.

Proof. Since $\Omega(r)-\Omega(\hat{r}) \geqq 0, \forall r \in[\hat{r}, R]$, and the other terms in the integrand are positive we get from (3.13), (3.4), and (3.12) that

$$
\Delta \theta^{\prime}(\varphi)>\frac{4}{\Omega(\hat{r})}\left[\frac{R^{2}-l^{2}}{h(R)-l^{2}}\right]^{1 / 2}>\frac{4}{\Omega(R)}=2 .
$$

Note that by (3.12), $\Omega(r) \in(0,2), \forall r \in(0, R)$, is equivalent to $V$ being attracting.

Corollary 5.2. Any attracting potential $V(r)$ satisfying the assumptions of Theorem 5.1 will produce a flow $\left(\phi^{t}, \mathscr{M}, \mu\right)$ for which the maximal Lyapunov exponents $\lambda^{+}(x)$ are positive almost everywhere and is ergodic.

Proof. If $V$ is $C^{2}$, then $\Omega$ is $C^{1}$ and we show in Appendix II that the Lyapunov exponents exist. Then combining Theorem 2.1, Theorem 4.2 and Theorem 5.1 proves the result. Ergodicity is discussed in Sect. 6.

From $\Omega$ we can determine the behavior of $V$ at $r=0$.

Lemma 5.3. Let $\Omega \in C^{1}([0, R])$ and $\alpha=2-\Omega(0)$. Then $V$ will have a singularity at $r=0$ of the form $-1 / r^{\alpha}$. 

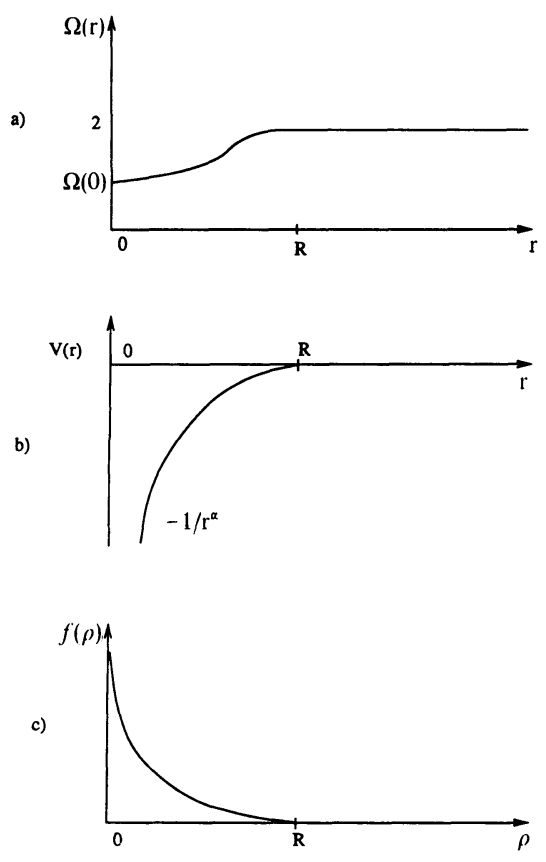

Fig. 5. Attracting Potential

Proof. Using (5.1), we get that

$$
\lim _{r \rightarrow 0} V(r)\left(\frac{r}{R}\right)^{2-\Omega(0)}=\text { constant }-\frac{1}{2} \exp \left(\int_{0}^{R} \frac{\Omega(0)-\Omega(s)}{s} d s\right) .
$$

The integral in (5.2) converges, for $\Omega \in C^{1}$, proving the lemma.

Example. For any $\alpha \in(0,2)$, we can construct smooth potentials $V(r)$ with singularity of order $-r^{-\alpha}$ for which the flow has positive Lyapunov exponents almost everywhere and is ergodic: take $\Omega$ monotone increasing in (5.1) with $\Omega(0)=2-\delta$ and $\Omega(R)=2, \Omega^{(k)}(R)=0, \forall k \geqq 1$ (this proves Theorem 1 ). For such a potential, we use (7.5) to show the relationship between $\Omega$ and $V$ (Fig. 5a, b). For these potentials, the associated Maupertius metric can be isometrically embedded in $\mathbb{R}^{3}$ as a surface of revolution determined by a function $z=f(\rho), \rho \in[0, R]$ (see Appendix V). We graph this function in Fig. 5c.

Remark. 5.4. If we consider a smooth attracting potential with no singularity at the origin and for which the rotation function is continuous, we are unable to prove positive Lyapunov exponents.

To see this, note that if $V$ is smooth at the boundary then $\lim \Delta \theta^{\prime}(\varphi)=2$ since for $\varphi=0$ the particle is tangent to the disk and hence stays in the $V \equiv 0$ region. And we know that for $V \equiv 0, \Delta \theta^{\prime}(0)=2$. So we are forced to make $\Delta \theta^{\prime}>2$ for all $\varphi$, since if $\Delta \theta^{\prime}$ decreases continuously from 2 the cone method breaks down 
(see Remark 4.7). But if $V$ is smooth and bounded, and $\Delta \theta \in C^{1}$ then

$$
\Delta \theta(\pi / 2)=\pi=\int_{0}^{\pi / 2} \Delta \theta^{\prime}(\varphi) d \varphi
$$

so that $\Delta \theta^{\prime}$ cannot be always bigger than 2 .

Regularization. Knauf [Kn1] showed that for potentials with singularities of the type $-r^{-2(1-1 / n)}, n \in Z^{+} \backslash\{0,1\}$, the flow could be regularized in an appropriate covering space, i.e. could be extended to a smooth flow. We explain why the regularization is possible using the rotation function $\Delta \theta$.

\section{Lemma 5.5 .}

$$
\lim _{\varphi \rightarrow \pi / 2^{-}} \Delta \theta(\varphi)=2 \pi / \Omega(0) .
$$

Proof. We introduce the function

$$
\Gamma(\lambda)=1 / \Omega\left(h^{-1}\left(\lambda^{2}\right)\right), \quad \lambda \in[-R, R],
$$

where $h^{-1}$ is $h$ inverse. Then the change of variables $h(r)=\lambda^{2}$ applied to (3.7) yields for $\varphi \in(0, \pi / 2)$.

$$
\begin{gathered}
\Delta \theta(\varphi)=4 \int_{1}^{R / l} \frac{\Gamma(l \lambda)}{\lambda \sqrt{\lambda^{2}-1}} d \lambda, \\
l=R \cos (\varphi)=h(\hat{r})=\text { angular momentum. }
\end{gathered}
$$

Taking the limit as $l \rightarrow 0$ and using that $\Gamma(0)=1 / \Omega(0), \int_{1}^{\infty} \frac{d \lambda}{\lambda \sqrt{\lambda^{2}-1}}=\pi / 2$ gives the
result. For $\Omega(0)=\frac{2}{n}, n>1, n \in Z$, one has that $\lim _{\varphi \rightarrow \pi / 2^{-}} \Delta \theta(\varphi)=n \pi$. In these cases, we can use (3.11) to define a rotation function continuous at $\pi / 2$. In fact, using (5.5) one can show that the rotation function is smooth at $\pi / 2$. The degree of smoothnes depends on the smoothness of $\Omega$. Thus for these examples, we could define $\Delta \theta(\pi / 2)$ and $t_{0}(\pi / 2)$ to produce a smooth system and hence an ergodic flow (see Sect. 6).

II. Repelling Potentials. Sinai [Si1] and Kubo [Ku] have given examples of repelling potentials, $V(r) \geqq 0, V^{\prime}(r) \leqq 0, r \in(0, R)$, for which the system had positive entropy and was ergodic. In their examples, the potential was continuous but not $C^{1}$.

We prove positive Lyapunov exponents for a class of repelling potentials which generalizes Kubo's "bell-shaped” potentials.

The reason that one has not yet been able to make $C^{1}$ potentials for which one can prove positive Lyapunov exponents can be understood by reference to Remark (4.7). If the potential is smooth, then $\Delta \theta^{\prime}(0)=2$. For small angles, the repelling nature of the potential causes trajectories to rotate less far around the disk than they would in the $V \equiv 0$ case. Hence for small $\varphi, \Delta \theta^{\prime}(\varphi)<2$. Thus the values of $\Delta \theta^{\prime}$ fill up some interval [2- $\left.-\delta, 2\right]$, and our cone-field method can not handle this case. 
Theorem 5.6. If $V$ satisfies conditions (3.2), (3.6) and

i. $\Omega(r)$ is non-increasing for $r \in(0, R)$.

ii. $V\left(R^{-}\right)=0$.

iii. $V^{\prime}\left(R^{-}\right)<0$.

Then

$$
\Delta \theta^{\prime}(\varphi) \leqq 2-\delta, \quad \forall \varphi \in(0, \pi), \text { for some } \delta>0 .
$$

Proof. $\Omega(r)$ is non-increasing, hence we have $\Omega(r)-\Omega(\hat{r}) \leqq 0, \forall r \in[\hat{r}, R)$, while the other terms in the integrand (3.13) are all positive. Since $\Omega(\hat{r})^{-1} \leqq \Omega\left(R^{-}\right)^{-1}$, we get

$$
\Delta \theta^{\prime}(\varphi) \leqq 4 \Omega\left(R^{-}\right)^{-1}\left[\frac{R^{2}-l^{2}}{h\left(R^{-}\right)-l^{2}}\right]^{1 / 2} \leqq \frac{4}{\Omega\left(R^{-}\right)}<2,
$$

using that (ii) implies $h\left(R^{-}\right)=R^{2}$ and (ii), (iii) imply by (3.12) that $\Omega\left(R^{-}\right)>2$.

Remark 5.7. The previous theorem holds for more general conditions than (ii), (iii). See example (II.b) below.

Using Theorem 4.3. (Remark 4.4.) and estimate (5.7) gives

Corollary 5.8. Any repelling potential $V(r)$ satisfying the assumptions of Theorem 5.6 and for which $t_{\min }$ satisfies $t_{\min }>\frac{4 R}{\Omega\left(R^{-}\right)-2}$ will produce a flow $\left(\varphi^{t}, \mathscr{M}, \mu\right)$ for which the maximal Lyapunov exponents $\lambda^{+}(x)$ are positive almost everywhere and is ergodic. Proof. Theorem 4.3 and Theorem 5.6 implies that $\lambda^{+}(x) \geqq 0$ a.e. while the ergodicity is discussed in Sect. 6.

Examples.

(II.a) Let $V(r)$ satisfy (3.2), (3.6) with

$$
\begin{aligned}
\Omega^{\prime}(r) \leqq 0, \quad \forall r \in(0, R), \quad V(R) & =0, \\
V^{\prime}\left(R^{-}\right) & <0
\end{aligned}
$$

(see Fig. 6). Then by (3.12), $\Omega\left(R^{-}\right)=2\left(1-V^{\prime}\left(R^{-}\right) R\right)$, so that

$$
\Delta \theta^{\prime}(\varphi) \leqq \frac{2}{1-V^{\prime}\left(R^{-}\right) R}<2, \forall \varphi \in[0, \pi] .
$$

For such a potential, Corollary 5.8 gives that $t_{\min }$ should satisfy

$$
t_{\min }>-\frac{2}{V^{\prime}\left(R^{-}\right)}
$$

Kubo $[\mathrm{Ku}]$ showed that if the potential was "bell-shaped", i.e.

$$
\frac{d}{d r}\left(-r V^{\prime}(r)\right) \leqq 0, \quad V(R)=0
$$

and if

$$
-V\left(R^{-}\right)>\frac{2}{R},
$$


a)

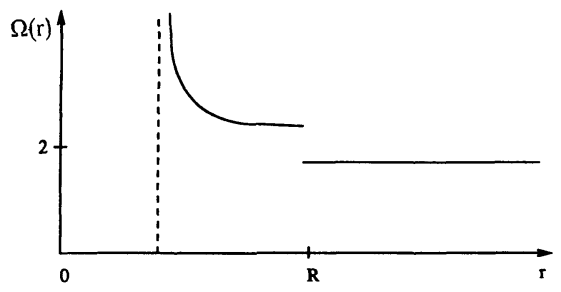

b)

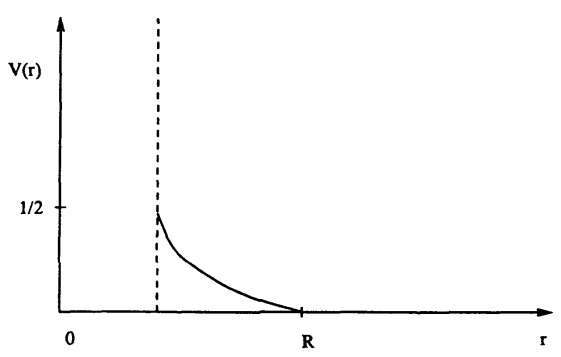

Fig. 6. Repelling Potential

then the system would be ergodic and have positive Lyapunov exponents almost everywhere, for $t_{\min }$ sufficiently large. A direct calculation, and the relation $1-2 V(r)>0$, shows that (5.11a) implies $\Omega^{\prime}(r) \leqq 0$. Thus our condition (5.8a) is weaker than Kubo's. Note that Kubo needs that $R V^{\prime}\left(R^{-}\right)<-2(5.11 \mathrm{~b})$ while we allow any $R V^{\prime}\left(R^{-}\right)<0(5.8 \mathrm{~b})$ (i.e. any arbitrary small corner for $V$ at the boundary). Also Kubo's minimum time, $t_{\min }>\frac{2 R}{-R V^{\prime}\left(R^{-}\right)-2}$, is larger than ours (5.10).

(II.b) We can allow discontinuities for $V$ at the boundary: $V\left(R^{-}\right) \neq 0$. If we choose $V(r) \equiv V_{0}, r \in\left[0, R^{-}\right)$, we have the case of a soft potential studied by Knauf [Kn2]; his results on positive Lyapunov exponents follows from our general set up. (Note that he normalizes the energy to be 1 instead of $1 / 2$ as we do.) For such a $V$,

$$
\Delta \theta^{\prime}(\varphi)=2\left[\frac{R^{2}-l^{2}}{h\left(R^{-}\right)-l^{2}}\right]^{1 / 2}
$$

When $V_{0}<0$ then

$$
\Delta \theta^{\prime}(\varphi) \leqq \frac{2}{\sqrt{1-2 V_{0}}}<2
$$

so we get positive Lyapunov exponents provided that $t_{\min }>2 R\left[\sqrt{1-2 V_{0}}-1\right]^{-1}$. The situation for $V_{0} \in(0,1 / 2)$ is slightly different. For $(R \cos \varphi)^{2}=l^{2} \geqq h\left(R^{-}\right)$, we have $\Delta \theta(\varphi) \equiv 0$. For $l^{2}<h\left(R^{-}\right),(5.12)$ gives $\Delta \theta^{\prime}>2$. Hence soft potentials produce positive Lyapunov exponents by two different mechanisms. Clearly one can produce examples where $V\left(R^{-}\right) \neq 0$ and $V$ is not constant provided that $\Omega$ has the appropriate behavior.

III. Mixed Potentials. A potential $V(r)$ being smooth does not necessarily imply that the rotation function $\Delta \theta(\varphi), \varphi \in[0, \pi / 2)$, is smooth. It is this observation that 
allows us to construct smooth, bounded potentials for which the flow has positive Lyapunov exponents and is ergodic. This idea was originally introduced in [D1].

The potential we construct will have a closed orbit for some $r_{c}<R$, hence $\Omega\left(r_{c}\right)=0$. There will be an angle $\varphi_{c} \in(0, \pi / 2)$ of entry for which the trajectory will become asymptotic to this closed trajectory and hence never leave the disk. Thus $\Delta \theta\left(\varphi_{c}\right)$ will be undefined.

Theorem 5.9. There exist smooth potentials, satisfying (3.2), (3.6), with a closed orbit at some $r_{0}<R$ for which the rotation function satisfies

$$
\begin{gathered}
\Delta \theta^{\prime}(\varphi)>2, \quad \varphi \in\left(0, \varphi_{c}\right), \\
\lim _{\varphi \rightarrow \varphi_{c}}\left|\Delta \theta^{\prime}(\varphi)\right|=\infty, \\
\Delta \theta^{\prime}(\varphi)<2-\delta, \quad \varphi \in\left(\varphi_{c}, \pi / 2\right], \quad \delta>0 .
\end{gathered}
$$

By introducing the closed orbit, we permit the rotation function to start out with $\Delta \theta^{\prime} \geqq 2$ for small angles and then to have $\Delta \theta^{\prime} \leqq 2-\delta$ for larger angles. Yet $\Delta \theta^{\prime}$ never takes values in the interval $(2-\delta, 2)$.

The theorem is proved in Appendix III. The potential we construct will staisfy

$$
\begin{aligned}
& \Omega(R)=2, \\
& \Omega(r) \geqq 0, \quad r \in[0, R], \\
& \Omega^{\prime}(r) \geqq 0, \quad r \in\left(r_{c}, R\right], \\
& \Omega^{\prime}\left(r_{c}\right)=0, \quad \Omega\left(r_{c}\right)=0, \\
& \Omega^{\prime} \leqq 0, \quad r \in\left[0, r_{c}\right),
\end{aligned}
$$

plus some additional technical conditions (Fig. 7).

Combining the previous Theorem and the results of Sect. 3 on positive Lyapunov exponents and Sect. 6 on ergodicity gives

Corollary 5.10. For a potential whose rotation function satisfies (5.13) and for which $t_{\min }>2 R(2-\delta) / \delta$, the flow has positive Lyapunov exponents almost everywhere and is ergodic.

Remark 5.11. There exist smooth potentials for which (5.13) holds that have a hard core: i.e. for some $r^{*}<r_{c}$, the potential satisfies $V(r)>1 / 2$ for all $r \in\left(0, r^{*}\right)$ (Fig. 8). The particle can not enter this region. For such systems, the associated geodesic flow is incomplete and therefore the [BG1] proof of ergodicity does not apply.

IV. Positive Entropy but Non-Ergodic Potentials. We outline the proof that there exist potentials whose flow has positive entropy but is not ergodic (Theorem 4).

We take a potential with $\Omega(r)<0$ for $r \in\left(r_{1}, r_{c}\right)$, with $\Omega\left(r_{1}\right)=\Omega\left(r_{c}\right)=0$ and with $0<r_{1}<r_{c}<R$ (Fig. 9). For such a potential the annulus between $r_{1}$ and $r_{c}$ is a trapping zone: there is a positive measure set of trajectories that stay forever in this region. These trajectories lie on invariant tori in phase space and hence have zero Lyapunov exponent. These trajectories prevent the system from being ergodic.

All trajectories that enter the disk with angle $\varphi \neq \varphi_{c}$ will leave the disk. By 
a)

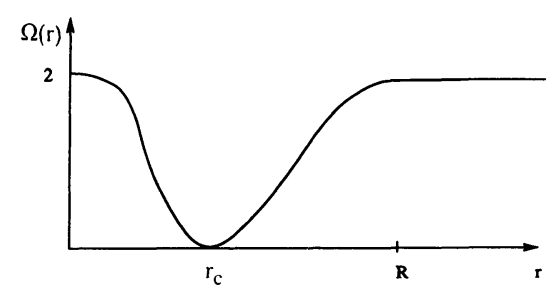

b)

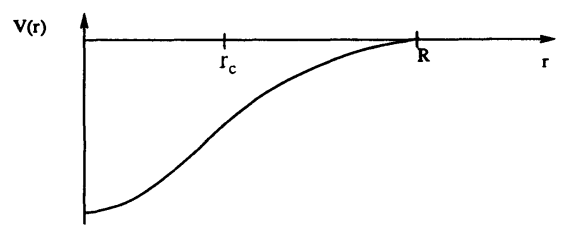

c)

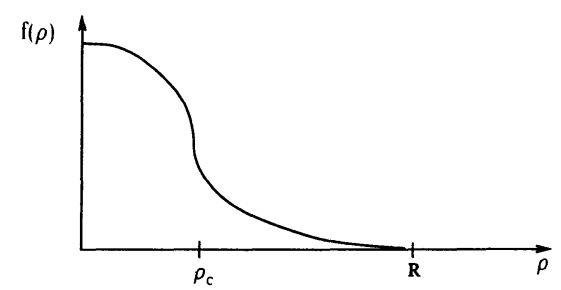

Fig. 7. Mixed Potential

a)

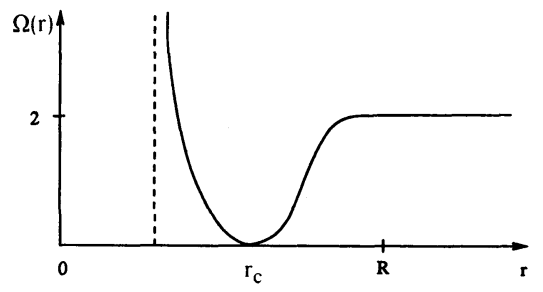

b)

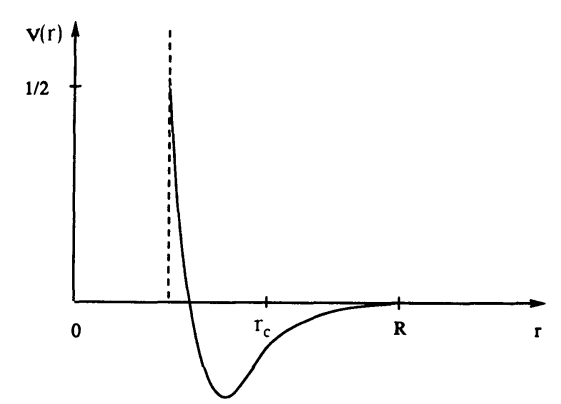

Fig. 8. Mixed Potential with incomplete Maupertius metric 


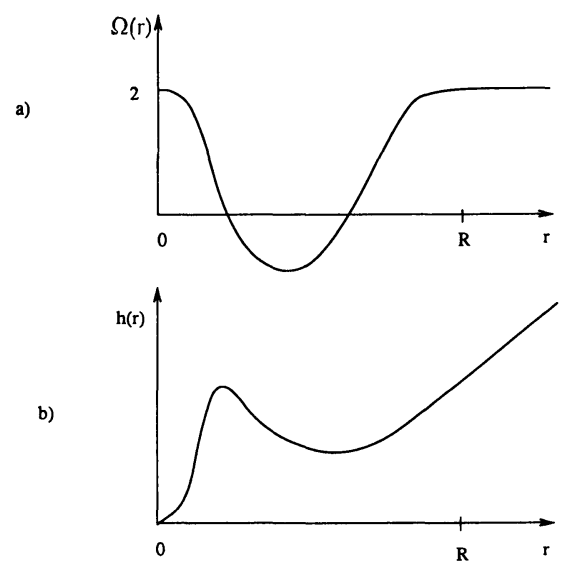

Fig. 9. Trapping Region

applying the methods of Theorem 5.9, we can choose $\Omega$ so that (5.13) holds. Then applying the cone-field argument to the invariant set of positive measure consisting of trajectories that enter the disk infinity often, we get that almost every such trajectory will have a positive Lyapunov exponent.

One can construct a positive entropy but non-ergodic potential in this way such that any symmetric perturbation of the potential retains these properties.

$V$. Varying the Energy Level. In our examples, we examined the $E=1 / 2$ subspace and produced potentials for which the flow restricted to the subspace would be ergodic. For such a potential, how does the flow behave on other energy levels?

If we take an attracting potential given by the example in I, then for any energy surface $E \geqq 1 / 2$, the flow restricted to that energy surface will have positive Lyapunov exponent almost everywhere and will be ergodic. For the repelling potentials given by example II.a, the same conclusion holds provided that $E \leqq 1 / 2$. For the mixed potentials, if $E>1 / 2$ then $\Omega(r)>0, r \in(0, R)$ and we are unable to determine the stochastic properties of the system. For $E<1 / 2$, the system will have a trapping zone and hence will not be ergodic. It is possible that these non-ergodic systems could have positive entropy as in IV.

All these results follow from repeating the analysis of Sect. 3 for the general case of energy $E$ rather than fo $E=1 / 2$. In Eq. (3.4), we replace $h(r)$ by

$$
h_{E}(r)=2 r^{2}(E-V(r)) \text {. }
$$

Then (3.12) holds with $\Omega(r)$ replaced by

$$
\Omega_{E}(r)=2-\frac{V^{\prime}(r) r}{E-V(r)}
$$

Thus for a fixed potential $V(r)$, the functions $\Omega=\Omega_{1 / 2}$ and $\Omega_{E}$ are related by

$$
\Omega_{E}=\Omega_{1 / 2}+\frac{\left(E-\frac{1}{2}\right)}{(E-V(r))} \frac{V^{\prime}(r) r}{\left(\frac{1}{2}-V(r)\right)},
$$


and hence

$$
\Omega_{E}^{\prime}(r)=\Omega_{1 / 2}^{\prime}(r)\left(\frac{\frac{1}{2}-V(r)}{E-V(r)}\right)+\left(E-\frac{1}{2}\right) \frac{V^{\prime}(r)\left(2-\Omega_{1 / 2}\right)}{(E-V(r))^{2}} .
$$

Thus for an attracting potential with $\Omega_{1 / 2}^{\prime}(r)>0$, if $E>1 / 2$, then $\Omega_{E}^{\prime}(r)>0$. Therefore the rotation function $\Delta \theta_{E}(\varphi)$ for the energy surface satisfies $\Delta \theta_{E}^{\prime}(\varphi)>2$. Similar arguments apply for the other cases.

\section{Ergodicity}

The method of Chernov and Siani [SC] was designed to prove ergodicity for maps with singularities. It may also be useful for smooth flows since such systems can have a non-smooth return map to a Poincaré section. All our ergodicity claims can be proven using this method (see Appendix IV).

For the smooth systems, there are simpler approaches to proving ergodicity, although no one method handles all the cases. We can use the method of [D2] to get ergodicity for the examples of Theorem 5.9. We can also appeal to the continuous cone-field criteria of Burns and Gerber [BG1, Theorem 1.1], but there is a slight problem. For our flow there does not exist an invariant two dimensional distribution $P$ :

$$
d \phi^{t} P(x)=P\left(\phi^{t} x\right)
$$

(i.e. the flow is not a contact flow). If such a distribution existed, outside the disk it would be given as the span of $\left\{X_{v^{\perp}}, X_{\Phi}\right\}$. Going through the disk, Lemma 4.5 shows that a vector in this subspace can pick up a component in the flow direction. A possible solution to the problem would be to prove a non-contact version of the Burns-Gerber theorem.

Instead, we will re-parameterize the flow in the disk so that

$$
R \Delta \theta^{\prime}(\varphi) \cos \varphi-\frac{d t_{0}}{d \varphi}(\varphi)=0,
$$

where $t_{0}(\varphi)$ is the time spent in the disk.

If the flow through the disk is given by a geodesic flow, then we know that this condition holds since geodesic flows are contact (the perpendicular subspace remains perpendicular).

Given a potential, we examine the geodesic flow of the associated Maupertius metric. If this flow is smooth and complete (Remark 5.11), then we can apply [BG1] to it. The trajectories of the geodesic flow and the potential flow are the same but are parameterized with different speeds. Since ergodicity is invariant under these reparametrizations, we get ergodicity for the potential flow.

For the attracting potentials with singularity of order $-1 / r^{\alpha}, \alpha=2(1-1 / n)$, $n \in Z^{+} \backslash\{0,1\}$ we showed that the flow was regularizable (Sect. 5). To prove ergodicity for these flows, we can prove ergodicity of a closely related system $\left(\mathscr{N}, \psi^{t}, v\right)$.

To construct $\mathscr{N}$, we start with the set $U$ (see Definition 4.1) and its boundaries 
$\mathscr{S}$ and $\tilde{\mathscr{S}}$. We will connect $\mathscr{S}$ and $\tilde{\mathscr{S}}$ by a suspended flow with height function

$$
t_{0}(\varphi)=\int_{0}^{\varphi} R \Delta \theta^{\prime}(s) \cos s d s \quad \varphi \in[0, \pi / 2] .
$$

Note that $t_{0}(\varphi)$ is bounded. We set.

$$
\Gamma=\left\{(x, t): x=(\theta, \phi) \in \mathscr{S}, t \in\left[0, t_{0}(\phi)\right]\right\}
$$

and identify the points $(x, 0),\left(x, t_{0}(\phi)\right)$ with the points $x \in \mathscr{S}$ and $\tilde{x}=(\theta+\Delta \theta(\phi),-\phi) \in \tilde{\mathscr{S}}$ respectively. Our space $\mathcal{N}$ is then $U \cup \Gamma$. The flow $\psi^{t}$ agrees with $\phi^{t}$ on $U$, while on $\Gamma, \psi^{t}\left((x, s)=(x, s+t)\right.$. The $\psi^{t}$-invariant measure $v$ is induced by $\mu$.

Our choice of $t_{0}(\varphi)$ implies that (6.1) holds. For $\varphi \in(0, \pi)$, the flow $\psi^{t}$ is smooth by the implicit function theorem (since $\Delta \theta^{\prime}(\varphi)$ is smooth). At $\varphi=0$, the flow is also smooth since as $\varphi \rightarrow 0, t_{0}(\varphi)$ behaves as it would in the $V \equiv 0$ case $\left(t_{0}(\varphi)=2 R \sin \varphi\right)$. Thus we can apply [BG1] to get ergodicity for $\psi^{t}$ and hence $\phi^{t}$.

For a potential of Theorem 5.9 whose geodesic flow is incomplete (Remark 5.11), we can prove ergodicity by combining the geodesic flow parameterization with the height function argument, provided we do the above construction in a disk contained inside the close orbit.

\section{Surfaces}

We derive relationships between the potentials discussed in Sect. 5 and their associated metrices given by (1.3).

For geodesic flow, the evolution of tangent vectors to phase space is determined by the Jacobi equation

$$
J^{\prime \prime}(t)+K(t) J(t)=0
$$

where $K(t)$ is the curvature along an orbit. Our equation (4.11) is just a special case of the Jacobi equation in the case $K=0$. The projective coordinate $u=J^{\prime} / J$ satisfies the Riccati equation

$$
u^{\prime}(t)=-K(t)-u^{2}(t)
$$

which is the generalization of (4.24). In negative curvature, one can easily see that solutions of (7.2) grow exponentially, and hence the maximal Lyapunov exponent is non-zero.

Lemma 7.1. For a given potential $V(r)$, the curvature of the associated metric ds is

$$
K(r)=-\frac{r \Omega^{\prime}(r)}{h(r)}
$$

so that

$$
\operatorname{sign}(K)=-\operatorname{sign}\left(\Omega^{\prime}\right) .
$$

Proof. For metrics conformal to the Euclidean metric, and of the form (1.3), one has that, [Kn1],

$$
K(q)=\frac{(E-V(q)) \Delta V(q)+(\nabla V(q))^{2}}{(E-V(q))^{3}}
$$


with the gradient $\nabla$ and the two-dimensional Laplacian $\Delta$. For $V$ radially symmetric, one has $\Delta V(r)=\left(\frac{\partial^{2}}{\partial r^{2}}+\frac{1}{r} \frac{\partial}{\partial r}\right) V(r)$ and $(\nabla V(r))^{2}=\left(\frac{\partial V}{\partial r}\right)^{2}$. From (3.12), we have

$$
V^{\prime}(r)=\frac{(2-\Omega(r))(1-2 V(r))}{2 r} .
$$

The result now follows by a straightforward calculation.

From this lemma, we see that the attractive potentials of Sect. 5 correspond to metrics of negative curvature. The repelling potentials correspond to metrics of positive curvature. The smooth potentials correspond to a mixed case in which a closed geodesic in zero curvature separates the two regimes.

\section{Appendix I. Rotation Function}

Let us remember that

$$
\begin{aligned}
\Delta \theta(\varphi) & =2 \int_{1}^{R f^{-1}} \frac{l}{s \sqrt{h(\hat{r} s)-l^{2}}} d s, \\
h(\hat{r}) & =l^{2}=R^{2}(\cos \varphi)^{2} .
\end{aligned}
$$

Moreover we can restrict ourselves to the case $l \geqq 0, \hat{r}<R, h^{\prime}(\hat{r}) \neq 0$. Then

$$
\Delta \theta(\varphi)=2 \int_{1}^{R f^{-1}} s^{-1}[(h(\hat{r} s) / h(\hat{r}))-1]^{-1 / 2} d s
$$

We can now take the derivative with respect to $\varphi$,

$$
\begin{aligned}
\Delta \theta^{\prime}(\varphi)= & -2 \hat{r}^{-1}\left[\left(h\left(R^{-}\right) / h(\hat{r})\right)-1\right]^{-1 / 2} \frac{d \hat{r}}{d \varphi} \\
& -\int_{1}^{R \hat{r}^{-1}} s^{-1}[(h(\hat{r} s) / h(\hat{r}))-1]^{-3 / 2}\left[\frac{s h^{\prime}(\hat{r} s) h(\hat{r})-h^{\prime}(\hat{r}) h(\hat{r} s)}{h(\hat{r})^{2}}\right] \frac{d \hat{r}}{d \varphi} d s .
\end{aligned}
$$

Differentiating the second of the (I.1) we get

$$
h^{\prime}(\hat{r}) \frac{d \hat{r}}{d \varphi}=-2 l R \sin \varphi=-2 l \sqrt{R^{2}-l^{2}}
$$

Using the previous result we have

$$
\begin{aligned}
\Delta \theta^{\prime}(\varphi)= & 4\left[R^{2}-l^{2}\right]^{1 / 2}\left[h\left(R^{-}\right)-l^{2}\right]^{-1 / 2} \hat{r}^{-1} h^{\prime}(\hat{r})^{-1} h(\hat{r}) \\
& +2 \int_{1}^{R \hat{r}^{-1}}\left[h(\hat{r} s)-l^{2}\right]^{-3 / 2}\left[R^{2}-l^{2}\right]^{1 / 2} \frac{h(\hat{r}) h(\hat{r} s)}{\hat{r} h^{\prime}(\hat{r})}\left[\frac{\hat{r} s h^{\prime}(\hat{r} s)}{h(\hat{r} s)}-\frac{\hat{r} h^{\prime}(\hat{r})}{h(\hat{r})}\right] d s \\
= & 2 \Omega(\hat{r})^{-1}\left[R^{2}-l^{2}\right]^{1 / 2}\left\{2\left[h\left(R^{-}\right)-l^{2}\right]^{-1 / 2}-\int_{1}^{R \hat{r}^{-1}} \frac{h(\hat{r} s)[\Omega(\hat{r} s)-\Omega(\hat{r})]}{s\left[h(\hat{r} s)-l^{2}\right]^{3 / 2}} d s\right\} .
\end{aligned}
$$

Formula (3.13) is then obtained by a simple change of variable in the integral. 


\section{Appendix II. Existence of the Lyapunov Exponents}

In this appendix we provide the estimates needed to prove the existence of the Lyapunov exponents for the flows considered in the paper. In particular we study the case when the potential is singular at the origin (Theorem 5.1). The existence of the Lyapunov exponents in the other cases follows from standard considerations. Let us remember that existence of the Lyapunov exponents is granted by condition (2.1). In order to verify (2.1) we need some explicit estimates.

Note that

$$
\begin{aligned}
h(r) & =r^{2}(1-2 V(r)), \\
\Omega(r) & =\frac{h^{\prime}(r) r}{h(r)} \\
l & =h(r)^{1 / 2} \cos \varphi .
\end{aligned}
$$

The equations of motion are given by

$$
\begin{aligned}
\dot{r}(t) & =r(t)^{-1} h(r(t))^{1 / 2} \sin \varphi(t), \\
\dot{\varphi}(t) & =\frac{\Omega(r(t))}{2 r(t)^{2}} l, \\
\dot{\theta}(t) & =\operatorname{lr}(t)^{-2}, \\
r(0) & =r_{0} ; \quad \varphi(0)=\varphi_{0} ; \quad \theta(0)=\theta_{0} .
\end{aligned}
$$

From (II.2) it is possible to explicitly compute $d \phi^{t}$ in polar coordinates and check that condition (2.1) is satisfied. Here we will explicitly find a bound for $\frac{\partial \varphi(t)}{\partial \varphi(0)}$; similar or easier estimates apply to the other elements of the Jacobian of $\phi^{t}$.

Lemma II.1. Given $T>0$ we have

$$
\begin{aligned}
\frac{\partial \varphi(t)}{\partial \varphi_{0}}= & 1-l \tan \varphi_{0} \int_{r_{0}}^{r(t)} \frac{1}{\rho}\left\{\frac{\Omega(\rho)}{2}+\frac{\Omega^{\prime}(\rho) \rho}{\Omega(\rho)}-2\right\} \frac{1}{\sqrt{h(\rho)-l^{2}}} d \rho \\
& +\int_{r_{0}}^{r(t)} \frac{1}{\rho}\left\{\frac{\Omega^{\prime}(\rho) \rho}{\Omega(\rho)}-2\right\} \frac{\partial \varphi}{\partial \varphi_{0}}\left(r^{-1}(\rho)\right) d \rho \quad \text { if } \quad \dot{r}(t) \neq 0 \forall t \in[0, T] .
\end{aligned}
$$

Proof. From (II.2) we have

$$
\varphi(t)=\varphi_{0}+\int_{0}^{t} \frac{\Omega(r(s))}{2 r(s)^{2}} l d s
$$

Differentiating the above expression, after some computations we get

$$
\frac{\partial \varphi}{\partial \varphi_{0}}=1-l \tan \varphi_{0} \int_{0}^{t} \frac{\Omega(r(s))}{2 r(s)^{2}} d s+l \int_{0}^{t} r(s)^{-3}\left\{\frac{1}{2} \Omega^{\prime}(r(s)) r(s)-\Omega(r(s))\right\} \frac{\partial r(s)}{\partial \varphi_{0}} d s .
$$

In order to have a closed equation we need to express $\frac{\partial r}{\partial \varphi_{0}}$ in terms of $\frac{\partial \varphi}{\partial \varphi_{0}}$. To 
do so we differentiate the last of the (II.1) with respect to $\varphi_{0}$ and obtain

$$
\frac{\partial r}{\partial \varphi_{0}}=-\tan \varphi_{0} \frac{2 r}{\Omega(r)}+\frac{2 r^{2}}{\Omega(r) l} \dot{r} \frac{\partial \varphi}{\partial \varphi_{0}},
$$

where we have used the first of (II.2). Using the above expression and changing variables in the integral the lemma is proven.

Using the preceding lemma we can obtain an interesting integral inequality.

Lemma II.2. Let $\dot{r}(t) \neq 0 \forall t \in[0, T]$. Then $\exists C_{3}, C_{4} \in \mathbb{R}^{+}$such that

$$
\left|\frac{\partial \varphi(t)}{\partial \varphi_{0}}\right| \leqq 1+C_{3}\left|\tan \varphi_{0}\right|+C_{4} \int_{r(t)}^{r_{0}} \frac{1}{\rho}\left|\frac{\partial \varphi\left(r^{-1}(\rho)\right)}{\partial \varphi_{0}}\right| d \rho .
$$

Proof. We will consider the case $r(t)<r_{0}$; the other case can be treated in the same way. Conditions (3.2), (3.6) imply $\Omega \in C^{1}\left(\mathbb{R}^{+}\right)$and, according to Theorem 5.1, $\Omega(r) \geqq \Omega(0)>0$; so

$$
\begin{aligned}
& \left|\int_{r_{0}}^{r(t)} \frac{1}{\rho}\left\{\frac{\Omega(\rho)}{2}+\frac{\Omega^{\prime}(\rho) \rho}{\Omega(\rho)}-2\right\} \frac{1}{\sqrt{h(l)-l^{2}}} d \rho\right| \\
& \leqq C_{1} \int_{r(t)}^{r_{0}} \frac{1}{\rho \sqrt{h(l)-l^{2}}} d \rho=C_{1} l^{-1} \int_{h(r(t)) l^{-2}}^{h\left(r_{0}\right) l^{-1}} \frac{1}{\Omega\left(h^{-1}\left(\zeta l^{2}\right)\right) \zeta \sqrt{\zeta-1}} d \zeta \\
& \leqq C_{2} l^{-1} \int_{h(r(t)) l^{-2}}^{h\left(r_{0}\right) l^{-2}} \frac{1}{\zeta \sqrt{\zeta-1}} d \zeta \\
& =2 C_{2} l^{-1}|\arctan [\sqrt{\zeta-1}]|_{h(r(t)) l^{-2}}^{h\left(r_{0}\right) l^{-2}} \leqq 2 \pi C_{2} l^{-1}=C_{3} l^{-1} \text {. }
\end{aligned}
$$

The lemma follows from the above estimate.

To obtain a bound for $\frac{\partial \varphi}{\partial \varphi_{0}}$ we need the following technical lemma.

Lemma II.3. If $x(r)$ satisfies the integral inequality

$$
|x(r)| \leqq A+B \int_{r}^{r_{0}} \frac{1}{\rho}|x(\rho)| d \rho \quad \forall r_{0}>r>0 ; \quad A, B \in \mathbb{R}^{+},
$$

then

$$
\|x\|_{r} \leqq A\left(\frac{r_{0}}{r}\right)^{B}
$$

where

$$
\|x\|_{r}=\sup _{\rho \in\left[r, r_{0}\right]}|x(\rho)| .
$$

Proof. The first step is to prove, by induction, the following

$$
\|x\|_{r} \leqq A \sum_{K=0}^{N-1} \frac{1}{K !}\left[B \log \frac{r_{0}}{r}\right]^{K}+\frac{1}{N !}\left[B \log \frac{r_{0}}{r}\right]^{N}\|x\|_{r} ; \quad N \geqq 1 .
$$


For $N=1$ it is trivially true. Let us suppose it is true for $N$ then

$$
\begin{aligned}
\|x\|_{r} & \leqq A+B \int_{r}^{r_{0}} \frac{1}{\rho}\left\{A \sum_{K=0}^{N-1} \frac{1}{K !}\left[B \log \frac{r_{0}}{\rho}\right]^{K}+\frac{1}{N !}\left[B \log \frac{r_{0}}{\rho}\right]^{N}\|x\|_{\rho}\right\} d \rho \\
& =A+B \int_{0}^{\log \left(r_{0} / r\right)}\left\{A \sum_{K=0}^{N-1} \frac{1}{K !} B^{K} \zeta^{K}+\frac{1}{N !} B^{N} \zeta^{N}\|x\|_{r}\right\} d \zeta \\
& \leqq A \sum_{K=0}^{N-1} \frac{1}{K !}\left[B \log \frac{r_{0}}{r}\right]^{K}+\frac{1}{(N+1) !}\left[B \log \frac{r_{0}}{r}\right]^{N+1}\|x\|_{r} .
\end{aligned}
$$

Now for each $r$ there exists $N: \frac{\left[B \log r_{0} / r\right]^{N}}{N !}<1$ which implies that $\|x\|_{r}$ is bounded. The result follows then taking the limit $N \rightarrow \infty$.

We are now able to state the announced result.

Lemma II.4. If the conditions of Theorem 5.1 holds then for each $T \sup _{t \in[0, T]} \log \left|\frac{\partial \varphi(t)}{\partial \varphi_{0}}\right| \epsilon$ $L^{1}(\mathscr{M})$.

Proof. From Lemmas II.2, II.3 we obtain that for $l \neq 0$,

$$
\begin{aligned}
\sup _{t \in[0, T]} \log \left|\frac{\partial \varphi(t)}{\partial \varphi_{0}}\right| & \leqq \sup _{r \geqq r_{\text {min }}} \log \left\|\frac{\partial \varphi}{\partial \varphi_{0}}\right\|_{r} \leqq \sup _{r \geqq r_{\text {min }}} \log \left[\left(1+C_{3}\left|\tan \varphi_{0}\right|\right)\left(\frac{r_{0}}{r}\right)^{C^{4}}\right] \\
& =\log \left(1+C_{3}\left|\tan \varphi_{0}\right|\right)+C_{4} \log \left(\frac{r_{0}}{r_{\text {min }}}\right),
\end{aligned}
$$

where $r_{\min }$ is defined by the equation $h\left(r_{\min }\right)=l^{2}$. Given the relation between $h$ and $\Omega$ we have that $r_{\min }>C_{5} r_{0}\left[\cos \varphi_{0}\right]^{-(2 / \Omega(0))}$ which implies

$$
\sup _{t \in[0, T]} \log \left|\frac{\partial \varphi(t)}{\partial \varphi_{0}}\right| \leqq \frac{C_{6}}{\Omega(0)} \log \left[\cos \varphi_{0}\right]^{-1} \in L^{1}(\mathscr{M})
$$

with respect to the measure $r_{0} d r_{0} d \theta_{0} d \varphi_{0}$.

Finally the result follows from similar estimates on the other elements of the Jacobian and from the fact that the norm in polar coordinates does not introduce further divergences.

\section{Appendix III. Mixed Potentials}

In addition to conditions $(5.14 \mathrm{a}-\mathrm{e})$, we also require that the potential satisfy

$$
\frac{d}{d r}\left\{\frac{\left[h\left(r_{c}\right)-h(r)\right]^{1 / 2}}{\Omega(r)}\right\} \geqq 0, \quad r \in\left(r_{p}, r_{c}\right)
$$

where $0 \leqq r_{p}<r_{c}<R$, and

$$
4 h\left(r_{p}\right)<h\left(r_{c}\right) \Omega\left(r_{p}\right)^{2} .
$$

We will show that (5.13) holds for a potential satisfying $(5.14 \mathrm{a}-\mathrm{g})$. These 
conditions are not intended to be optimal and there are other ways to construct potentials satisfying (5.13) (see Alternative Construction).

Conditions (5.14c,e) imply that $\Omega^{\prime \prime} \geqq 0$ in some neighborhood of $r_{c}$, which in turn implies that $(5.14 \mathrm{f})$ holds in some interval $\left[r_{p}, r_{c}\right]$. Condition $(5.14 \mathrm{~g})$ is a requirement that this interval be sufficiently large. (If we could take $r_{p}=0$, then $(5.14 \mathrm{~g})$ would hold, while $(5.14 \mathrm{~g})$ fails if $\left.r_{p}=r_{c}\right)$. Note that if $\Omega\left(r_{p}\right)>2$ (corresponding to a potential with a repelling part) $(5.14 \mathrm{~g})$ is always satisfied, since $h\left(r_{p}\right)<h\left(r_{c}\right)$. Our conditions generalize the non-increasing curvature condition that Burns and Gerber derived for a focusing cap in the case of geodesic flow [BG2]. We will relate the two situations in Lemma III.2.

Proof of Theorem 5.9. For $\hat{r} \geqq r_{c}$, the proof is identical to the case of Theorem 5.1. Next, we use that $h(R)=R^{2}, h(\hat{r})=l^{2}$ and (3.12), to rewrite (3.13), getting

$$
\Delta \theta^{\prime}(\varphi)=2 \Omega(\hat{r})^{-1}\left\{2+\left[R^{2}-l^{2}\right]^{1 / 2} \int_{\hat{f}}^{R} \frac{[\Omega(s)-\Omega(\hat{r})]}{\Omega(s)} \frac{h^{\prime}(s)}{[h(s)-h(\hat{r})]^{3 / 2}} d s\right\} .
$$

For $\hat{r} \in\left[r_{p}, r_{c}\right)$ an estimate can be obtained by dividing the integral into two parts:

$$
\begin{aligned}
& \int_{r_{c}}^{R} \frac{[\Omega(s)-\Omega(\hat{r})]}{\Omega(s)} \frac{h^{\prime}(s)}{[h(s)-h(\hat{r})]^{3 / 2}} d s \\
& \quad \leqq \frac{2-\Omega(\hat{r})}{2} \int_{r_{c}}^{R} \frac{h^{\prime}(s)}{[h(s)-h(\hat{r})]^{3 / 2}} d s \\
& =(2-\Omega(\hat{r}))\left\{\left[h\left(r_{c}\right)-h(\hat{r})\right]^{-1 / 2}-\left[R^{2}-l^{2}\right]^{-1 / 2}\right\},
\end{aligned}
$$

and

$$
\begin{aligned}
& \int_{\hat{r}}^{r_{c}} \frac{[\Omega(s)-\Omega(\hat{r})]}{\Omega(s)} \frac{h^{\prime}(s)}{[h(s)-h(\hat{r})]^{3 / 2}} d s \\
&=\int_{\hat{f}}^{r_{c}}\left\{1-\frac{\Omega(\hat{r})}{\left[h\left(r_{c}\right)-h(s)\right]^{1 / 2}} \frac{\left[h\left(r_{c}\right)-h(s)\right]^{1 / 2}}{\Omega(s)}\right\} \frac{h^{\prime}(s)}{[h(s)-h(\hat{r})]^{3 / 2}} d s,
\end{aligned}
$$

which by condition $(5.14 \mathrm{f})$

$$
\leqq \int_{\hat{r}}^{r_{c}}\left\{1-\frac{\left[h\left(r_{c}\right)-h(\hat{r})\right]^{1 / 2}}{\left[h\left(r_{c}\right)-h(s)\right]^{1 / 2}}\right\} \frac{h^{\prime}(s)}{[h(s)-h(\hat{r})]^{3 / 2}} d s .
$$

Changing variables to $u=\frac{h(s)-h(\hat{r})}{h\left(r_{c}\right)-h(\hat{r})}$ we obtain

$$
\begin{aligned}
& =\frac{1}{\left[h\left(r_{c}\right)-h(\hat{r})\right]^{1 / 2}} \int_{0}^{1}\left\{1-\frac{1}{\sqrt{1-u}}\right\} u^{-3 / 2} d u \\
& =-\frac{2}{\left[h\left(r_{c}\right)-h(\hat{r})\right]^{1 / 2}} \int_{0}^{\pi / 2}\left\{1-\frac{1}{\cos \theta}\right\} \frac{\cos \theta}{\sin ^{2} \theta} d \theta=-\frac{2}{\left[h\left(r_{c}\right)-h(\hat{r})\right]^{1 / 2}} .
\end{aligned}
$$

Combining this estimate and (III.2), we get

$$
\Delta \theta^{\prime}(\varphi) \leqq 2 \Omega(\hat{r})^{-1}\left[R^{2}-l^{2}\right]^{1 / 2}\left\{\frac{2}{\left[h\left(r_{c}\right)-h(\hat{r})\right]}-\frac{2}{\left[h\left(r_{c}\right)-h(\hat{r})\right]}\right\}=0 .
$$


For $\hat{r} \in\left[0, r_{p}\right]$ it is necessary to divide the interval of integration in (III.1) in three parts, namely $\left[r_{c}, R\right],\left[r_{p}, r_{c}\right]$ and $\left[\hat{r}, r_{p}\right]$. Estimate (III.2) applies for the first interval. The second interval can be treated according to (III.3) yielding

$$
\begin{aligned}
\int_{r_{p}}^{r_{c}} \frac{\Omega(s)-\Omega(\hat{r})}{\Omega(s)} \frac{h^{\prime}(s)}{[h(s)-h(\hat{r})]^{3 / 2}} d s \leqq & 2\left[h\left(r_{c}\right)-h(\hat{r})\right]^{-1 / 2}\left\{-1+\left[\frac{h\left(r_{c}\right)-h(\hat{r})}{h\left(r_{p}\right)-h(\hat{r})}\right]^{1 / 2}\right. \\
& \left.-\frac{\Omega(\hat{r})}{\Omega\left(r_{p}\right)} \frac{h\left(r_{c}\right)-h\left(r_{p}\right)}{\left[h\left(r_{c}\right)-h(\hat{r})\right]^{1 / 2}\left[h\left(r_{p}\right)-h(\hat{r})\right]^{1 / 2}}\right\},
\end{aligned}
$$

using $\Omega(\hat{r}) \geqq \Omega\left(r_{p}\right)$, by $(5.14 \mathrm{e})$,

$$
\leqq 2\left[h\left(r_{c}\right)-h(\hat{r})\right]^{-1 / 2}\left\{-1+\left[\frac{h\left(r_{p}\right)-h(\hat{r})}{h\left(r_{c}\right)-h(\hat{r})}\right]^{1 / 2}\right\} .
$$

The contribution of the third interval is negative, due to (5.14e), in analogy with Theorem 5.6. Combining the previous estimates give

$$
\begin{aligned}
\Delta \theta^{\prime}(\varphi) \leqq & 2-2\left[R^{2}-l^{2}\right]^{1 / 2}\left\{\left[h\left(r_{c}\right)-h(\hat{r})\right]^{-1 / 2}-2 \Omega(\hat{r})^{-1} \frac{\left[h\left(r_{p}\right)-h(\hat{r})\right]^{1 / 2}}{h\left(r_{c}\right)-h(\hat{r})}\right\} \\
= & 2-2\left[R^{2}-l^{2}\right]^{1 / 2}\left[h\left(r_{c}\right)-h(\hat{r})\right]^{-1} \Omega\left(r_{p}\right)^{-1} \\
& \cdot\left\{\left[h\left(r_{c}\right)-h(\hat{r})\right]^{1 / 2} \Omega\left(r_{p}\right)-2\left[h\left(r_{p}\right)-h(\hat{r})\right]^{1 / 2}\right\} .
\end{aligned}
$$

According to (III.5) the theorem is proven provided that

$$
\left[h\left(r_{c}\right)-h(\hat{r})\right] \Omega\left(r_{p}\right)^{2}>4\left[h\left(r_{p}\right)-h(\hat{r})\right]
$$

which is implied by $(5.14 \mathrm{~g})$.

Remark III.1. If condition (5.14f) holds for all $r<r_{c}$, then the previous estimates imply that $\Delta \theta^{\prime}(\varphi)<0$ for all $\varphi \in\left(\varphi_{c}, \pi / 2\right]$.

Burns and Gerber [BG2] showed that if a Riemannian metric inside the disk had a closed orbit at $r_{c}$, was of negative Gaussian curvature $K(r)<0, r \in\left(r_{c}, R\right)$, and satisfied $\frac{d K(r)}{d r}<0$ for all $r \in\left(0, r_{c}\right)$, then the rotation function would satisfy $\Delta \theta^{\prime}(\varphi)<0$, $\varphi \in\left(\varphi_{c}, \pi / 2\right]$. Our condition (5.14f) is a generalization of their result.

Lemma III.2. Let $K(r)$ be the curvature of the Maupertius metric. If

$$
\frac{d K(r)}{d r}<0 \forall r<r_{c}
$$

then (5.14f) holds for all $r<r_{c}$.

Proof. Using identities (3.12), (7.3) and explicit computation yields

$$
\frac{d}{d r}\left\{\frac{\left[h\left(r_{c}\right)-h(r)\right]^{1 / 2}}{\Omega(r)}\right\}=-\frac{g(r) h(r)}{2 r \Omega(r)^{2}\left[h\left(r_{c}\right)-h(r)\right]^{1 / 2}},
$$

where

$$
g(r)=\Omega(r)^{2}-2 K(r)\left[h\left(r_{c}\right)-h(r)\right] .
$$


Accordingly

Using (3.12), and (7.3) again,

$$
\operatorname{sign}\left(\frac{d}{d r}\left\{\frac{\left[h\left(r_{c}\right)-h(r)\right]^{1 / 2}}{\Omega(r)}\right\}\right)=-\operatorname{sign}(g(r)) .
$$

$$
g^{\prime}(r)=-2 K^{\prime}(r)\left[h\left(r_{c}\right)-h(r)\right]>0
$$

and, since $g\left(r_{c}\right)=0$, this implies that $g(r)<0$ for all $r<r_{c}$.

Alternative Construction of Potential. Another way to produce a potential that will satisfy (5.13) is as follows (see [D1, Sect. 9] for the analogous construction in the geodesic flow case). Let $\Omega(r)>0, r \in\left(0, r_{c}\right)$ be an arbitrary smooth function satisfying (5.14d) with $\Omega^{\prime \prime}\left(r_{c}\right)>0$. Then there exists a value of $R$ and a way to define $\Omega(r)$, $r \in\left(r_{c}, R\right)$ such that $\Delta \theta^{\prime}(\varphi)<0$ for all $\varphi \in\left(\varphi_{c}, \pi / 2\right]$. To achieve this, let $\Omega$ satisfy $(5.14 \mathrm{a}, \mathrm{c})$ and make $\Omega$ very close to zero for a long interval after $r_{c}$. Such a choice of $\Omega$ will cause the contribution from the integral in (III.2) to become large negative and outweigh the possibly positive, but bounded, contribution from (III.4).

\section{Appendix IV. Ergodicity for Systems with Singularity}

We use the methods of [SC] as modified by [KSS3] (see also [Bu]) to prove ergodicity for the flows discussed in the paper. We will prove ergodicity of a return map $T$ to a Poincaré section $\mathscr{S}_{1}$ from which ergodicity of the flow $\phi^{t}$ will follow.

We define the cross-section $\mathscr{S}_{1}$ as $\mathscr{S} \cup\left\{(q, p) \mid \pi_{1}(q)=-1 / 2\right\} \cup\left\{(q, p) \mid \pi_{2}(q)=\right.$ $-1 / 2\}$, where $\mathscr{S}$ is given in Sect. 3 and $\pi_{i}(q)$ is the $i$ coordinate of $q$. We assume that the torus is a square of width one centered at the origin. By augmenting the original cross-section $\mathscr{S}$ we insure that the return time to the cross-section is finite. We equip $\mathscr{S}_{1}$ with the measure $\mu_{1}$ induced by the Liouville measure and a metric induced by the Riemannian metric. Furthermore we induce two cone fields $C^{+}$and $C^{-}$on $\mathscr{S}_{1}$. For $x \in \mathscr{S}, C^{+}(x)$ is the projection of the cone $C(x)$, defined in Sèct. 4, onto the tangent space $\mathscr{T}_{x} \mathscr{S}$. For $x \in \mathscr{S}_{1} \backslash \mathscr{S}, C^{+}(x)$ is defined by pushing forward the cones: if $y=T^{k} x$ for $x \in \mathscr{S}$ and $k$ minimum, then we set $C(y)=D T^{k}(C(x))$. The cone $C^{-}(x)$ is defined to be the closure of the complement of $C^{+}(x)$.

In all the cases under consideration we assume that the stable and unstable manifolds exist almost everywhere in $\mathscr{S}_{1}$ and that these manifolds have the property of absolute continuity. If the original flow was smooth, then these results follows from Pesin theory [P]; if the flow has singularities, we refer to [KS]. Before proceeding further we introduce some additional notations.

\section{Definition IV.1.}

(1)

$$
\begin{aligned}
\mathscr{R}^{+} & =\left\{x \in \mathscr{S}_{1}: T \text { it is not smooth at } x\right\}, \\
\mathscr{R}^{-} & =\left\{x \in \mathscr{S}_{1}: T^{-1} \text { it is not smooth at } x\right\}, \\
\mathscr{R} & =\mathscr{R}^{+} \cup \mathscr{R}^{-} .
\end{aligned}
$$

(2) A point $x \in \mathscr{S}_{1}$ is sufficient for $T$ if there exists $n(x)$ such that

i) $T^{k}(x) \notin \mathscr{R}^{+}, \quad k \in\{0,1,2, \ldots, n(x)-1\}$,

ii) $D T^{n(x)}\left(C^{+}(x)\right) \subset \operatorname{Int}\left(C^{+}\left(T^{n(x)} x\right)\right)$. 
(3) A point $x \in \mathscr{S}_{1}$ is sufficient for $T^{-1}$ if the analogous of properties (i), (ii) holds for the complementary cone field $C^{-}$and the set $\mathscr{R}^{-}$.

Since our system has positive Lyapunov exponents almost everywhere, Pesin theory [P], in the version by Katok and Strelcyn [KS], implies that for the maps $T$ under consideration the set $\mathscr{S}_{1}$ is the union (modulo a set of zero measure) of a countable number of ergodic components. The next theorem categorizes the points that belong to an ergodic component with a nice local structure (non-empty interior).

Theorem IV.2 (Local Ergodicity). Choose $x \in \mathscr{S}_{1}$ for which $T^{k}$ is smooth for all $k \neq 0$. If $x$ is sufficient for both $T$ and $T^{-1}$ then there exists a neighborhood $\mathscr{U}(x)$ of $x$ that belongs to one ergodic component.

To prove global ergodicity we need first a simple extension of Theorem IV.2.

Theorem IV.3 (Crossing Singularity Lines). Choose $x \in T^{-n} \mathscr{R}^{+}\left(T^{n} \mathscr{R}^{-}\right)$for which $T^{-k} x\left(T^{k} x\right)$ is smooth for all $k>0$. If $x$ is sufficient for $T^{-1}(T)$ then $T^{-n} \mathscr{R}^{+}\left(T^{n} \mathscr{R}^{-}\right)$ cannot be the boundary between two different ergodic components.

Global ergodicity then follows from an analysis of the set of non-sufficient points.

Theorem IV.4 (Global Ergodicity). If the set of non-sufficient points for $T$ and $T^{-1}$ does not separate $\mathscr{S}_{1}$ then there is only one ergodic component.

Note that sufficiency is equivalent to a trajectory entering the disk. The only trajectories that do not enter the disk are periodic orbits that move on the torus with a rational angle. For a given rational angle, there will always be a non-empty set of trajectories that enter the disk, so the condition of Theorem IV.4 is satisfied. We will not discuss explicitly Theorem IV.3 since its proof is analogous to part of the proof of Theorem IV.2.

Proof of Theorem IV.2 ( A Sketch). We refer to [KSS3] for the details of the proof. Here we confine ourselves to a discussion of the changes needed to apply their results to our situation and how they fit into the general argument. The proof of [KSS3] requires the following properties.

Property I (Double Singularities). The set of double singularities of order $n$ (i.e. points that enter $\mathscr{R}^{+}$before $n$ forward iterates and also enter $\mathscr{R}^{-}$before $n$ backward iterates of $T$ ) is a finite union of isolated points.

Property II (Ansatz). Let $v$ be the measure induced by $\mu_{1}$ on $\mathscr{R}^{+} \cup \mathscr{R}^{-}$, then for $v$-almost every $y \in \mathscr{R}^{+}\left(\mathscr{R}^{-}\right)$the map $T^{-k}\left(T^{k}\right)$ is smooth for all $k>0$ and $y$ is sufficient for $T^{-1}(T)$.

Both properties can be proven using the same technique. First we notice that the singularity set $\mathscr{R}$ and the set of non-sufficient points are the union of a finite number of smooth manifolds. Then we study how the tangent vectors to these manifolds are situated with respect to the cone structure on $\mathscr{S}_{1}$.

We illustrate the argument in the case of an attracting potential. Let $\mathscr{R}_{1}^{+} \subset \mathscr{R}^{+}$ be the manifold of points that in future time hit the center of the potential. A tangent vector to the manifold $\mathscr{R}_{1}^{+}$gives rise to a variation of trajectories that is 
converging. Hence this tangent vector must lie in $C^{-}$. Analogously, the manifold $\mathscr{R}_{1}^{-} \subset \mathscr{R}^{-}$, of the trajectories that hit the singularity in the past has tangent vectors belonging to the cones $\mathrm{C}^{+}$. Given the invariance property of the cone families, any intersection of the manifolds $T^{-k} \mathscr{R}_{1}^{+}$and $T^{j} \mathscr{R}_{1}^{-}$(double singularities) is transversal (the tangent vectors to the manifolds belonging to complementary cones). Carrying out a similar analysis for all the singular points and non-sufficient points of our examples yields Properties I, II.

Property III (Expansion). The vectors inside the cones $C^{+}\left(C^{-}\right)$expand monotonically for $D T\left(D T^{-1}\right)$.

Here the expansion is measured by the induced metric on $\mathscr{S}_{1}$. The length of a vector $\xi_{1} \in \mathscr{T} \mathscr{S}_{1}$ in this induced metric is just the length of the vector $\xi \in \mathscr{T} \mathscr{M}$ in the original metric, where $\xi$ is the unique vector in the $\left\{X_{v^{\perp}}, X_{\varphi}\right\}$ plane which projects under the flow to $\xi_{1}$.

Hence to prove Property III for $T\left(T^{-1}\right)$, we study the corresponding property for the projected flow (i.e. flow under $\phi^{t}$ and then project onto the $\left\{X_{v^{\perp}}, X_{\varphi}\right\}$ plane). We split the analysis into two cases:

$$
\text { (1) } \Delta \theta^{\prime} \geqq 2 \text { or } \Delta \theta^{\prime} \leqq 0 .
$$

According to (4.12) the vector $\xi=J X_{v^{\perp}}+J^{\prime} X_{\varphi}$ is mapped into the vector

$$
\tilde{\xi}=\left(\begin{array}{c}
\tilde{J} \\
\tilde{J}^{\prime}
\end{array}\right)=\left(\begin{array}{c}
J\left(\Delta \theta^{\prime}-1\right)+J^{\prime}\left(R \Delta \theta^{\prime} \sin \varphi\right) \\
J \frac{\Delta \theta^{\prime}}{R \sin \varphi}+J^{\prime}\left(\Delta \theta^{\prime}-1\right)
\end{array}\right)
$$

from entering to leaving the disk. Thus for $\xi \in C(x)$ given by (4.2),

$$
\|\tilde{\xi}\|^{2} \geqq J^{2}\left(\Delta \theta^{\prime}-1\right)^{2}+J^{2}\left(\Delta \theta^{\prime}-1\right)^{2} \geqq J^{2}+J^{\prime 2}=\|\xi\|^{2} .
$$

And using (4.11), we get that outside the disk $\left\|D \phi^{t} \tilde{\xi}\right\| \geqq\|\tilde{\xi}\|$ which implies the result.

$$
\text { (2) } 0<\Delta \theta^{\prime} \leqq 2-\delta \text {, for some } \delta \in(0,2) \text {. }
$$

In this case we have to consider only vectors of the form $\xi=(1, u) u \in\left[0, u^{\text {top }}\right]$, $u^{\text {top }}=\frac{\delta}{R(2-\delta)}($ see (4.26)). Using (IV.1) and (4.11) it follows that the image of the vector $\xi$, from entering to entering the potential again, is

$$
\tilde{\xi}=\left(\begin{array}{c}
\left(\Delta \theta^{\prime}-1\right)+\frac{\Delta \theta^{\prime}-2}{R \sin \varphi} \tau+u\left[R \Delta \theta^{\prime} \sin \varphi+(\Delta \theta-1) \tau\right] \\
\frac{\Delta \theta^{\prime}-2}{R \sin \varphi}+u\left(\Delta \theta^{\prime}-1\right)
\end{array}\right)=\left(\begin{array}{c}
\tilde{J} \\
\tilde{J}^{\prime}
\end{array}\right),
$$

where $\tau$ is the time from existing the potential to entering again. According to the hypothesis of Theorem $4.3, \tau \geqq t_{\min }>\frac{2 R(2-\delta)}{\delta}$. Thus

$$
-\widetilde{J}^{\prime} \geqq \frac{\delta}{R \sin \varphi}+u(\delta-1) \geqq \frac{\delta}{R}+u(\delta-1)=u^{\mathrm{top}}(2-\delta)+u(\delta-1) \geqq u
$$


and

$$
-\widetilde{J} \geqq \delta-1+\frac{\delta}{R} t_{\min }+u\left[(\delta-2) R+(\delta-1) t_{\min }\right]>\delta-1+2(\delta-2)-\frac{(2-\delta)^{2}}{\delta} R u^{\text {top }}=1,
$$

which again implies $\|\tilde{\xi}\|>\|\xi\|$.

The last property requiring an independent proof in our cases is the following:

Property IV (Parallelization). The width of the cones goes monotonically to 0 .

Recalling (4.20), $\tilde{u}=f_{\varphi}(u)=\tilde{J}^{\prime} / \tilde{J}$, it is a direct computation to check that $\frac{d}{d u} f_{\varphi}(u)=(J / \widetilde{J})^{-2}$. Property IV is then a consequence of the above estimates.

This last property insures that the singularity lines become almost parallel to the (un)stable fibres and hence allows one to bound the measure of fibres that come close to the singularity lines.

Even if the unstable fibre, for example, through the point $T^{-k} x$ gets cut by a singularity line, and hence is short, it is still possible that the fiber through $x=T^{k}\left(T^{-k} x\right)$ is sufficiently long: one would need that the map $T^{k}$ expands the fiber sufficiently. This behavior can be achieved if at the singular point which cuts the fiber the map $D T^{k}$ is expansive.

Thus, as in [KSS3], we define

$$
k_{n, 0}^{+}(y)=\inf _{\xi \in C^{+}\left(T^{-n} n_{y}\right)}\left\|D T^{n} \xi\right\| .
$$

Then for $v$-almost all point $y \in \mathscr{R}^{-}$, we have $\lim _{n \rightarrow \infty} k_{n, 0}^{+}(y)=\infty$. This statement is a consequence of Property III, which implies that a sufficient point keeps expanding at least linearly (since this is the rate at which vectors in the interior of the cone expand outside of the potential, in the free motion) and, of Proposition I, which implies that almost every point in $\mathscr{R}^{-}$is sufficient.

Using all the previous information it is possible to apply [KSS3] to prove that the stable and unstable fiber are long enough to perform the standard Hopf $[\mathrm{H}]$ argument and prove ergodicity in a neighborhood of our original point $x \in \mathscr{S}_{1}$.

\section{Appendix V. Embedding}

Given a function $f(\rho), \rho \in[0, R]$, we define a surface of revolution as

$$
\left\{(x, y, z) \in R^{3}: x=\rho \cos \theta, y=\rho \sin \theta, z=f(\rho) \text { for } \theta \in[0,2 \pi), \rho \in[0, R]\right\} .
$$

The metric $d \sigma^{2}$ induced on this surface by the Euclidean metric on $R^{3}$ is

$$
d \sigma^{2}=\left(1+f^{\prime 2}(\rho)\right) d \rho^{2}+\rho^{2} d \theta^{2} .
$$

Suppose that our abstract metric $d s^{2}$ on the disk $D$ can be isometrically embedded in $R^{3}$ as a surface of revolution. Then there exists a monotone increasing function $g(r), r \in[0, R]$, with $g(0)=0, g(R)=R$ such that for $\rho=g(r)$ one has

$$
\begin{aligned}
\left(1+f^{\prime 2}(g(r))\right) g^{\prime 2}(r) d r^{2} & =(1-2 V(r)) d r^{2}, \\
g^{2}(r) d \theta^{2} & =(1-2 V(r)) r^{2} d \theta^{2} .
\end{aligned}
$$


Lemma V.1. The disk $D$ equipped with the metric $d s^{2}$ can be isometrically embedded in $R^{3}$ if

i. $|\Omega(r)| \leqq 2, \forall r \in[0, R]$,

ii. $\Omega(r) \geqq 0, \forall r \in[0, R]$, with equality holding at no more than a finite number of points, iii. $\lim _{r \rightarrow \infty} r^{2} V(r)=0$ and $V(R)=0$.

The functions $g$ and $f$ are given by

where

$$
\begin{aligned}
g(r) & =(h(r))^{1 / 2}, \\
f(\rho) & =f(g(r))=\int_{r}^{R} f^{\prime}(g(s)) g^{\prime}(s) d s,
\end{aligned}
$$

$$
f^{\prime}(g(r))=-\left(\frac{4}{\Omega^{2}(r)}-1\right)^{1 / 2} .
$$

Proof. We can solve (V.2b) by setting

$$
g(r)=r(1-2 V(r))^{1 / 2}=h^{1 / 2}(r) .
$$

Combining this with (iii) gives that $g(0)=0$ and that $g(R)=R$. Since $h^{\prime}(r)=$ $(h(r) \Omega(r)) / r$, (ii) implies that $g(r)$ is monotone increasing.

Using (V.3) in (V.2a) gives that

$$
\begin{aligned}
1+f^{\prime 2}(g(r)) & =\frac{r^{2}(1-2 V(r))}{r^{2} g^{\prime 2}(r)}=\frac{h(r)}{r^{2}}\left(\frac{2 g(r)}{h^{\prime}(r)}\right)^{2} \\
& =\frac{4}{\Omega^{2}(r)},
\end{aligned}
$$

so that (V.2a) is satisfied if

$$
f^{\prime}(g(\rho))=-\left(\frac{4}{\Omega^{2}(r)}-1\right)^{1 / 2}
$$

This equation is well defined if $|\Omega(r)| \leqq 2$. We define $f$ by $f(R)=0$ and $f(\rho)=$ $\int_{\rho}^{R} f^{\prime}(\rho) d \rho$.

Acknowledgements. The authors would like to thank L. Bunimovich, K. Burns, R. De La Llave, G. Gallavotti, M. Gerber, A. Knauf, D. Ruelle, and M. Wojtkowski for useful discussions. On the question of ergodicity, they profited greatly from discussions with A. Krámli, and D. Szász. They also thank the IMA. where part of this work was done.

\section{References}

[A] Arnold, V. I.: Mathematical methods of classical mechanics. Berlin, Heidelberg, New York: Springer 1978

[Ba] Baldwin, P. R.: Soft billiard systems. Physica D 29, 321-342 (1988)

[BC] Benedicks, M., Carleson, L.: The dynamics of the Hénon map, preprint

[Be] Berry, M.: Semiclassical mechanics of regular and irregular motion. Les Houches Session XXX. Amsterdam, New York, Oxford: North Holland 1981

[BG1] Burns, K., Gerber, M.: Continuous invariant cone families and ergodicity of flows in dimension three. Erg. Th. Dyn. Syst. 9, 19-25 (1989) 
[BG2] Burns, K. Gerber, M.: Real analytic Bernoulli geodesic flows on $S^{2}$. Erg. Th. Dyn. Syst. 9, 27-45 (1989)

[Bu] Bunimovich, L.A.: A Theorem on ergodicity of two-dimensional hyperbolic billiards. Commun. Math. Phys. 130, 599-621 (1990)

[D1] Donnay, V. J.: Geodesic flow on the two-sphere, part I: Positive measure entropy. Erg. Th. Dyn. Syst. 8, 531-553 (1988)

[D2] Donnay, V. J.: Geodesic flow on the two-sphere, part II: Ergodicity. Lecture Notes in Mathematics vol. 1342, pp 112-153. Berlin, Heidelberg, New York: Springer 1988

[G] Gallavotti, G.: Lectures on the Billiard. Lecture Notes in Physics vol. 38, pp. 236-295. Berlin, Heidelberg, New York: Springer 1975

[H] Hopf, E.: Statistik der Geodatischen Linien in Mannigfaltigkeiten Negativer Krummung. Leipziger Berichte 19, 261-304 (1939)

[K] Katok, A.: Invariant Cone families and stochastic properties of a smooth dynamical systems, preprint

[Kn1] Knauf, A.: Ergodic and topological properties of Coulombic periodic potentials. Commun. Math. Phys. 100, 89-112 (1987)

[Kn2] Knauf, A.: On soft billiard systems, Physica D36, 259-262 (1989)

[Kr] Krylov, N. S.: Works on the foundation of statistical physics. Princeton, NJ: Princeton University Press 1979

[KS] Katok, A., Strelcyn, J. M.: with collaboration of F. Ledrappier and F. Przytycki: Invariant Manifolds, Entropy and Billiards, Smooth Maps with Singularities. Lectures Notes in Mathematics vol. 1222. Berlin, Heidelberg, New York: Springer 1986

[KSS1] Krámli, A., Simányi, N., Szász, D.: Three billiard balls on the $v$-dimensional torus is a $K$-flow, submitted to Ann. Math.

[KSS2] Krámli, A., Simányi, N., Szász, D.: Four balls on the $v$-dimensional torus, preprint

[KSS3] Krámli, A., Simányi, N., Szász, D.: A transversal fundamental theorem for semi-dispersing billiards. Commun. Math. Phys. 129, 535-560 (1990)

[KSS4] Krámli, A., Simányi, N., Szász, D.: Dispersing billiards without focal points on surfaces are ergodic. Commun. Math. Phys. 125, 439-457 (1989)

[Ku] Kubo, I.: Perturbed Billiard Systems, I. The Ergodicity of the Motion of a Particle in a Compound Central Field. Nagoya Math. J. 61, 1-57 (1976)

[KuM] Kubo, I., Murata, H.: Perturbed Billiard Systems, II. Bernoulli Properties. Nagoya Math. J. 81, 1-25 (1981)

[M] Markarian, R.: Ergodic properties of a particle motion: Symmetric potentials; Non-elastic billiards. Preprint (1990)

[O] Osceledec, V. I.: A multiplicative ergodic theorem: Characteristic Lyapunov exponents of dynamical systems. Trans. Moscow Math. Soc. 19, 197-231 (1968)

[P] Pesin, Ya. B.: Lyapunov characteristic exponents and smooth ergodic theory. Russ. Math. Surv. 32, 4, 55-114 (1977)

[SC] Sinai, Ya. G., Chernov, N. I.: Ergodic properties of certain systems of two-dimensional discs and three-dimensional balls. Usp. Math. Nauk 42, 153-174 (1987)

[Si1] Sinai, Ya. G.: On the Foundations of the Ergodic Hypothesis for a Dynamical System of Statistical Mechanics, Dokl. Akad. Nauk SSSR 153, 1261-1264 (1963)

[Si2] Sinai, Ya. G.: Dynamical systems with elastic reflections. Russ. Math. Surv. 25(1), 137-189 (1970)

[V1] Vetier, A.: Sinai billiard in potential field (construction of fibers). Révész, P. (ed.). Coll. Math. Soc. J. Bolyai 36, 1079-1146 (1982)

[V2] Vetier, A.: Sinai billiard in potential field (absolute continuity) Proc. 3rd Pann. Symp. Mogyoródi, J., Vincze, I., Wertz, W. (eds.). 341-351 (1982)

[W1] Wojtkowski, M.: Invariant families of cones and Lyapunov exponents. Erg. Th. Dyn. Syst. 5, 145-161 (1985)

[W2] Wojtkowski, M.: A system of one dimensional balls with gravity. Commun. Math. Phys. 126, 507-533 (1990)

[W3] Wojtkowski, M.: The system of one dimensional balls in an external field II. Commun. Math. Phys. 127, 425-432 (1990) 